\title{
High Loading of Pd Nanoparticles by Interior functionalization of MOFs for Heterogeneous Catalysis
}

Bappaditya Gole, ${ }^{a}$ Udishnu Sanyal, ${ }^{a}$ Rahul Banerjee, ${ }^{b}$ Partha Sarathi Mukherjee ${ }^{a}$.

${ }^{a}$ Department of Inorganic and Physical Chemistry, Indian Institute of Science

Bangalore 560012, India

Fax: (+91)80-2360-1552; Tel: (+91)8o-2293-3352; E-mail:psm@ipc.iisc.ernet.in

${ }^{b}$ Physical and Materials Chemistry Division, CSIR-National Chemical

Laboratory, Dr. Homi Bhabha Road, Pune 411008, India

\section{Supporting Information}

\section{Experimental section}

1.1. Materials and methods

1.2. Single crystal $X$-ray diffraction analysis

2. Characterization of Heck-coupling reaction products

3. References

\section{Experimental section}

\subsection{Materials and methods}

All the chemicals/solvents were obtained from different commercial sources and used without further purification, unless otherwise mentioned. 5-(prop-2-yn-1-yloxy) isophthalic acid (pip) 
and 1,4-di(pyridin-4-yl)benzene $(d p b)$ were prepared according to the literature procedures. ${ }^{1}$ MOF-A was used for the control experiment which was synthesized according to our earlier report. ${ }^{2} \mathrm{NMR}$ spectra were recorded on a Bruker $400 \mathrm{MHz}$ spectrometer. The chemical shifts $(\delta)$ in the ${ }^{1} \mathrm{H}$ NMR spectra are reported in ppm relative to tetramethylsilane (TMS) as internal standard (0.0 ppm). IR spectra were recorded on a Bruker ALPHA FT-IR spectrometer used in the $4000-400 \mathrm{~cm}^{-1}$ range. Elemental analyses were carried out on Perkin Elmer $240 \mathrm{C}$ CHNS analyser. Powder X-ray diffraction (PXRD) patterns were recorded on a Phillips PANalytical diffractometer for $\mathrm{Cu} K \alpha$ radiation $(I=1.5406 \AA)$, with a scan speed of $1^{\circ} \mathrm{min}^{-1}$. Thermogravimetric (TGA) analyses of the MOFs were carried out on a Metler-Toledo thermal gravimetric analyzer under nitrogen flow. ESI-HRMS measurements were done on an Agilent 6538 UHD accurate-mass Q-TOF LC/MS instrument. Inductively coupled plasma atomic emission spectrometry (ICP-AES) was performed on a Shimadzu ICPS-8100 equipment. Field emission scanning electron microscopy (FESEM) was carried out using Carl-Zeiss Ultra 55 microscope equipped with energy dispersive X-ray analysis (EDX) at an operating voltage of 5$20 \mathrm{kV}$. EDX analysis was carried out to determine the elemental composition of the samples. Samples for SEM were prepared by drop casting the dispersion of samples in methanol onto pre-cleaned carbon tape. The TEM bright field (BF) images, selected area electron diffraction (SAED) patterns, high resolution TEM (HRTEM) images, and the high-angle annular dark field (HAADF) images were acquired using a JEOL 2100F field emission transmission electron microscope, both operating at $200 \mathrm{kV}$. To prepare the TEM samples, the metal nanopowder was first re-dispersed in acetone by sonicating for about $15 \mathrm{~min}$ and then $2 \mu \mathrm{L}$ of this solution was placed on a carbon coated copper grid and finally dried under a lamp.

\subsection{Single crystal $X$-ray diffraction analysis}

A Crystal of the MOF-2(Cd) was coated with paratone oil and the diffraction data were collected at $90 \mathrm{~K}$ on a Bruker SMART APEX CCD diffractometer using SMART/SAINT software. ${ }^{3}$ Intensity data were collected using graphite-monochromatized Mo-K $\alpha$ radiation (0.71073 $\AA$ ) at $90 \mathrm{~K}$. The structure was solved by direct methods using the SHELX-97 program ${ }^{4}$ incorporated into WinGX. ${ }^{5}$ Empirical absorption corrections were applied with SADABS. ${ }^{6}$ All non-hydrogen atoms were refined with anisotropic displacement coefficients. The hydrogen atoms bonded to carbon were included in geometric positions and given thermal parameters equivalent to 1.2 
times those of the atom to which they were attached. The hydrogen atoms of the solvent water molecule were not included in the least-squares refinement. Structure refinement after modification of the data for the solvent molecules with the SOUEEZE routine of PLATON led to better refinement and data convergence. The crystallographic refinement parameters are given in supporting information (Table S1). Several atoms of the solvent molecules are highly disordered and deviated from the ideal geometry which is mainly due to the high thermal vibration. This leads to a high $U_{\text {iso }}(\max ) / U_{\text {iso }}(\min )$ ratio for solvent atoms.

\begin{tabular}{|c|c|}
\hline & MOF- $2(\mathrm{Cd})$ \\
\hline Empirical formula & $\mathrm{C}_{23} \mathrm{H}_{18} \mathrm{O}_{5} \mathrm{~N}_{2} \mathrm{Cd}$ \\
\hline FW & 514.81 \\
\hline$T(\mathrm{~K})$ & $90(2)$ \\
\hline Crystal system & Monoclinic \\
\hline Space group & $C_{2} / c$ \\
\hline$a / \AA ̊$ & $29 \cdot 479(3)$ \\
\hline$b / \AA$ & $16.687(2)$ \\
\hline$c / \AA ̊$ & $15.766(2)$ \\
\hline$\alpha / \mathrm{deg}$ & 90 \\
\hline$\beta /$ deg & $113.530(5)$ \\
\hline$y / d e g$ & 90 \\
\hline$V / \AA^{3}$ & $7111.1(12)$ \\
\hline$Z$ & 4 \\
\hline$\rho_{\text {calcd }}\left(\mathrm{g} \mathrm{cm}^{-3}\right)$ & 1.383 \\
\hline$\mu(\mathrm{Mo} \mathrm{K} \alpha)\left(\mathrm{mm}^{-1}\right)$ & 0.669 \\
\hline$\lambda / \AA ̊$ & 0.71073 \\
\hline$F(000)$ & 3040.0 \\
\hline Collected reflns & 11023 \\
\hline Unique reflns & 5936 \\
\hline $\operatorname{GOF}\left(F^{2}\right)$ & 0.951 \\
\hline$R_{1}^{a}$ & 0.0934 \\
\hline$w R_{2}^{b}$ & 0.3267 \\
\hline
\end{tabular}



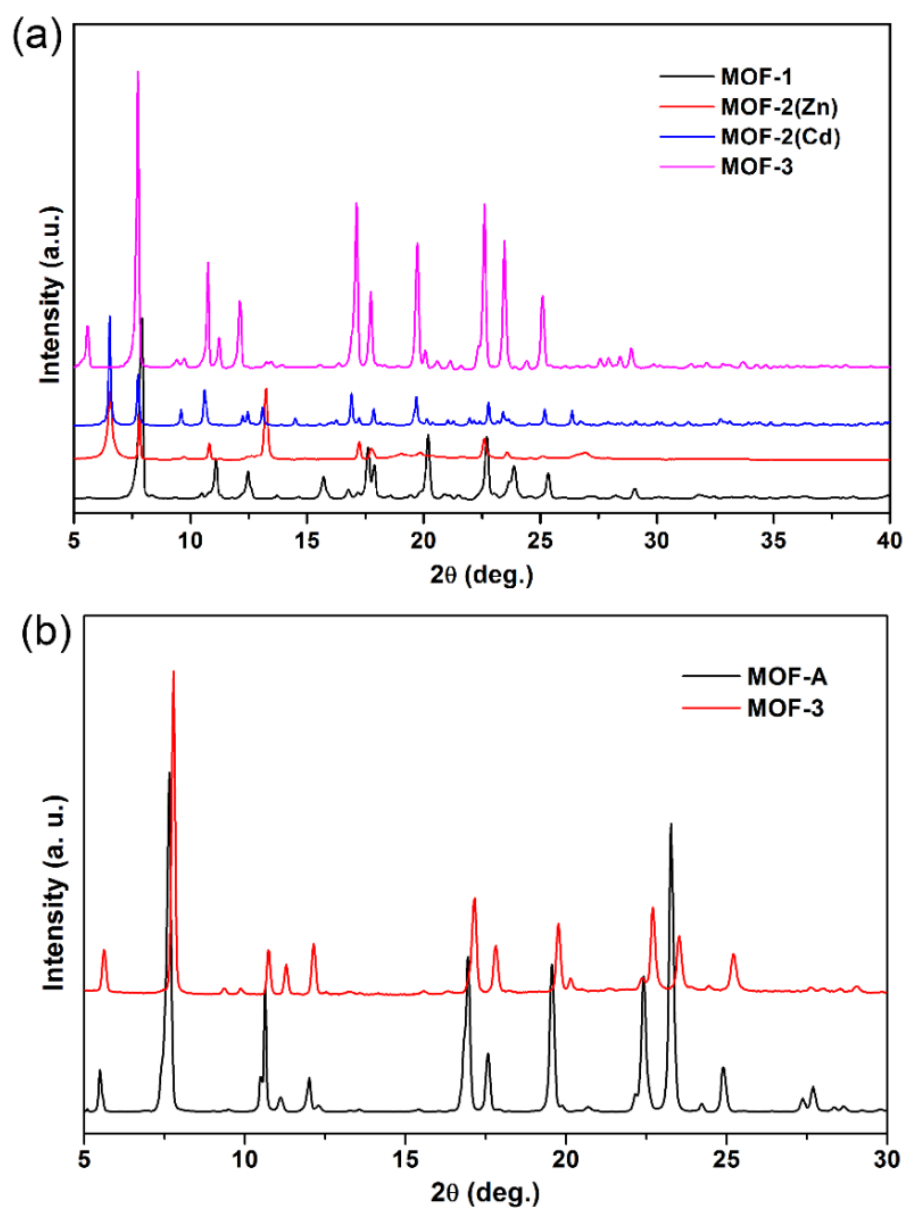

Figure S1. (a) PXRD patterns of as-synthesized MOFs. (b) Comparison of PXRD of MOF-3 and MOF-A which indicated that both adopt similar structure.

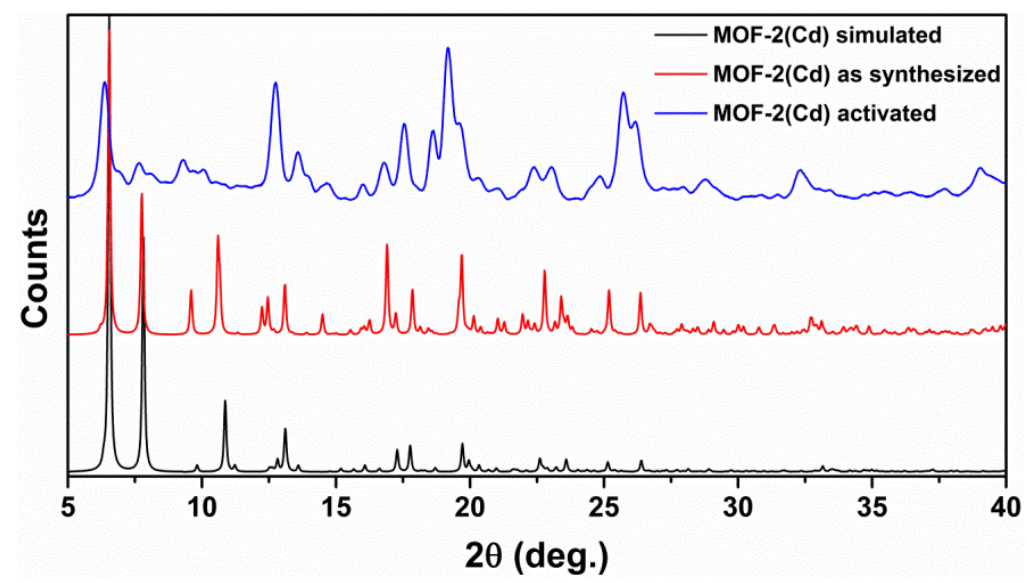

Figure S2: Comparison of simulated, as synthesized and activated PXRD patterns of different MOF-2(Cd).Similar comparison of the other MOFs were already reported in our earlier publication. ${ }^{7}$ 


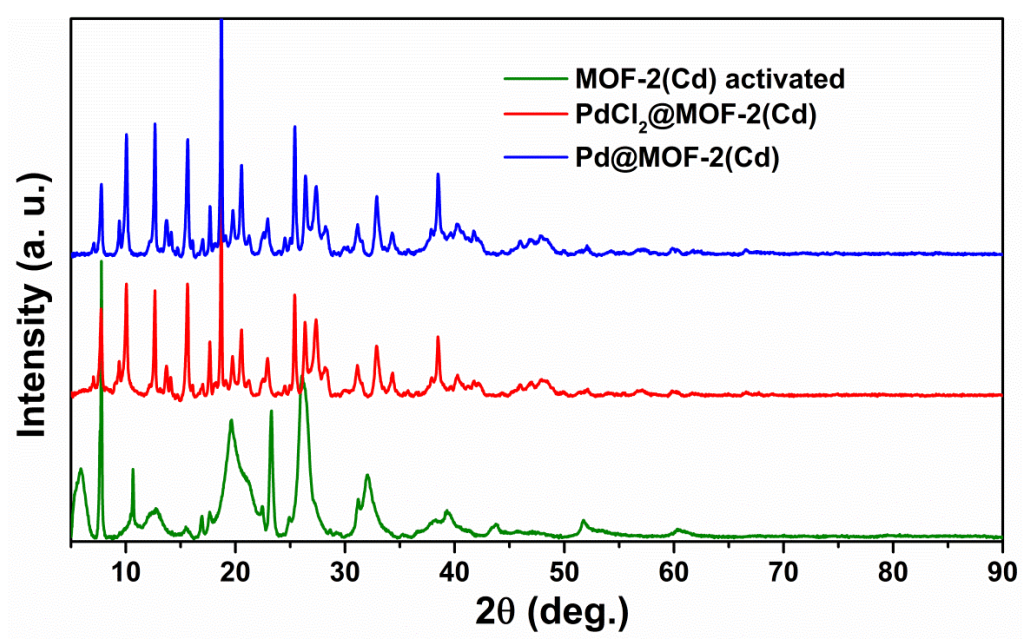

Figure S3. Comparison of PXRD pattern of MOF-2(Cd), PdCl $@$ @MOF-2(Cd) and Pd@MOF2(Cd) along with their visual color change after each step. The PXRD pattern of the activated sample of MOF-2(Cd) was observed very broad due to amorphous nature of the MOF after guest solvent removal. However, after adsorption of $\mathrm{PdCl}_{2}$, it appears extremely crystalline.

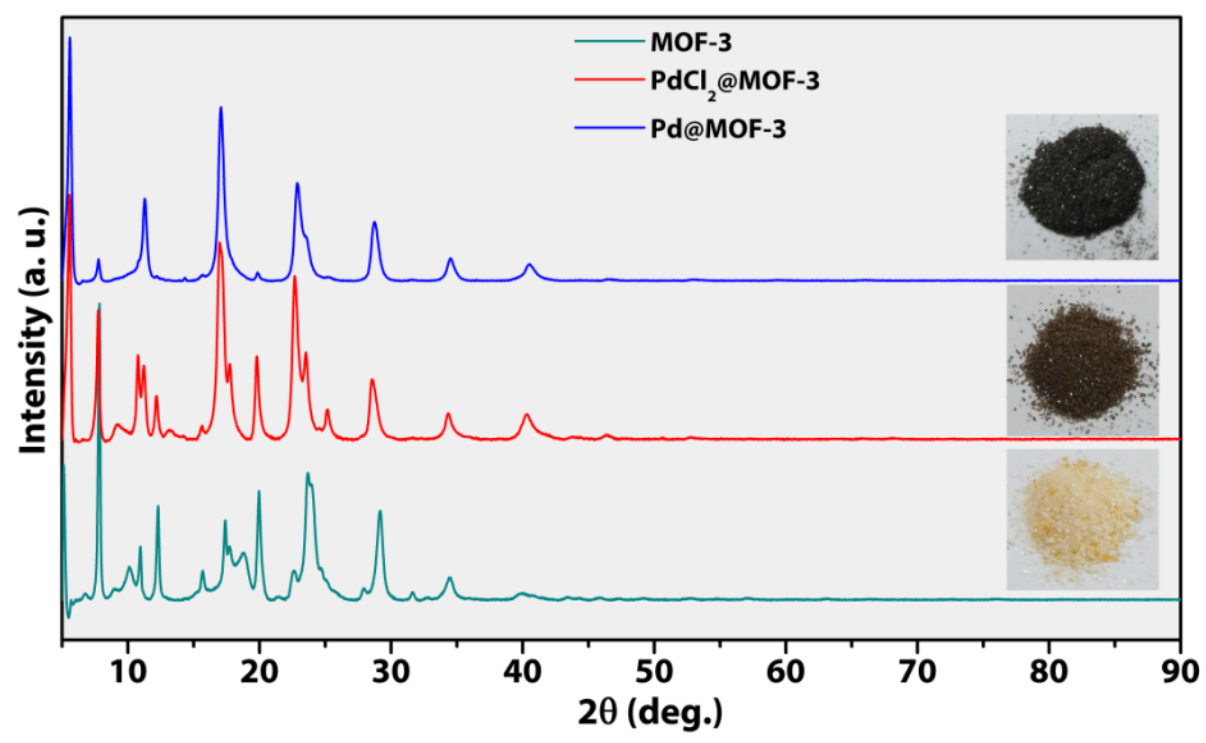

Figure S4. Comparison of PXRD pattern of MOF-3, PdCl $@$ @MOF-3 and Pd@MOF-3 along with their visual color change after each step. 

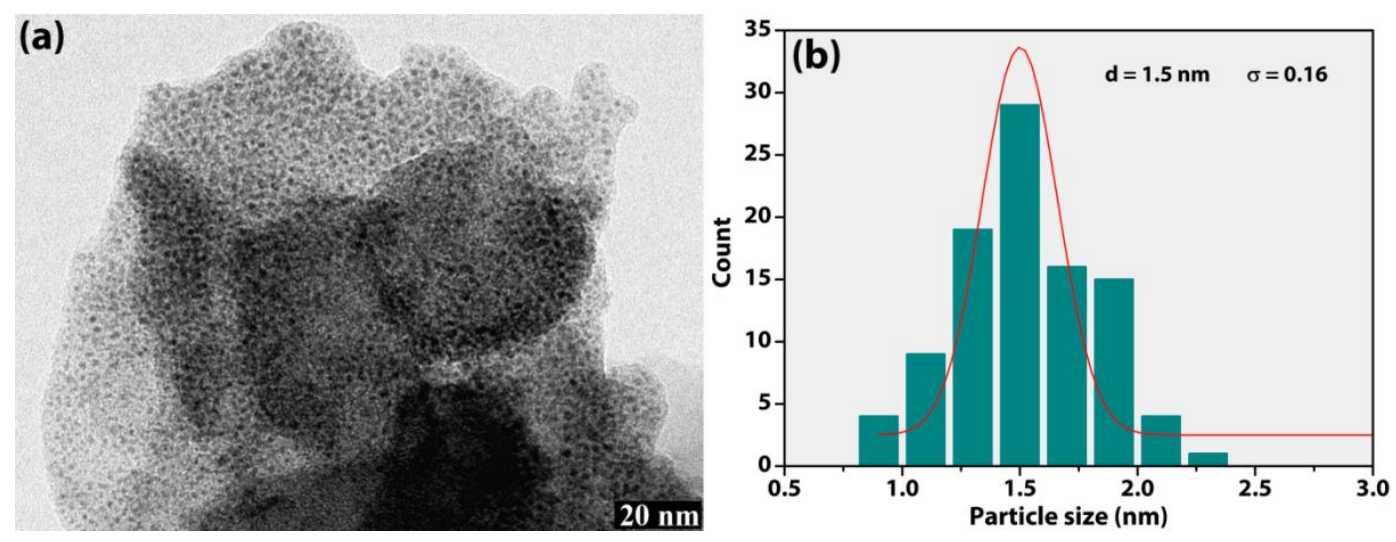

Figure S5. 10 wt \% Pd loading in MOF-1. (a) Bright-field TEM image showing the Pd NPs are homogeneously loaded in MOF-1. (b) Corresponding Pd NPs size distribution histogram.
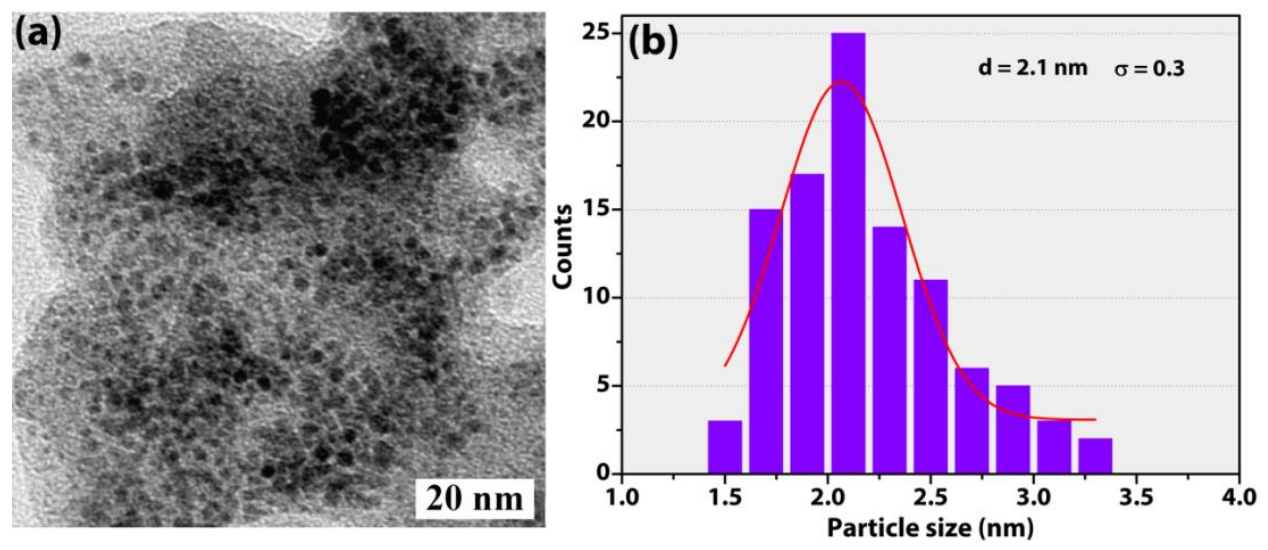

Figure S6. 12.5 wt \% Pd loading in MOF-2(Cd). (a) Bright-field TEM image showing the Pd NPs are homogeneously loaded in MOF-2(Cd). (b) Corresponding Pd NPs size distribution histogram.

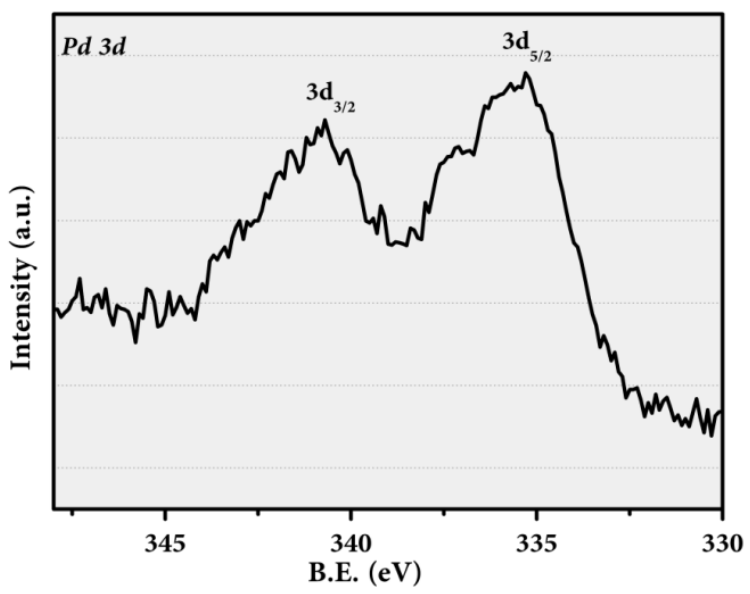

Figure S7. XPS spectrum of Pd@MOF-1 corresponds to $\mathrm{Pd}^{\circ} \mathrm{NPs}$. 


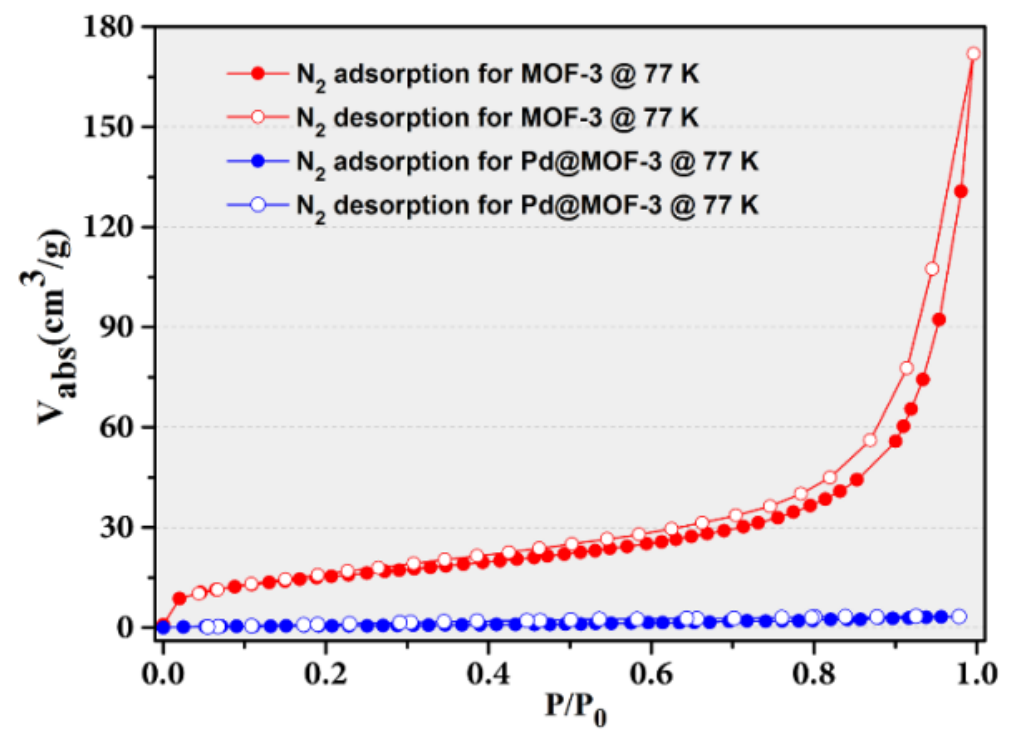

Figure S8. Nitrogen gas adsorption of MOF-3 and Pd@MOF-3 at $77 \mathrm{~K}$. The surface area reduced significantly after loading of Pd NPs into MOF-3 compared to MOF-3 itself.

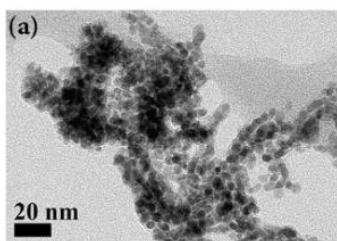

(c)

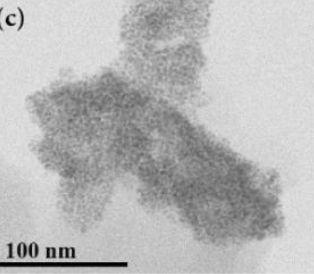

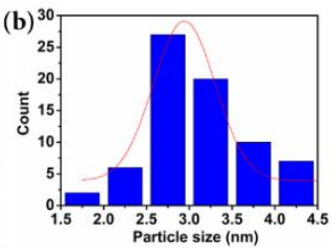

(d)

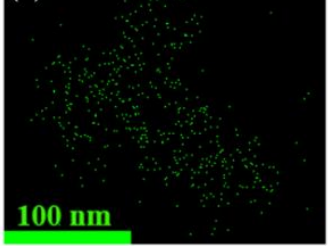

(e)

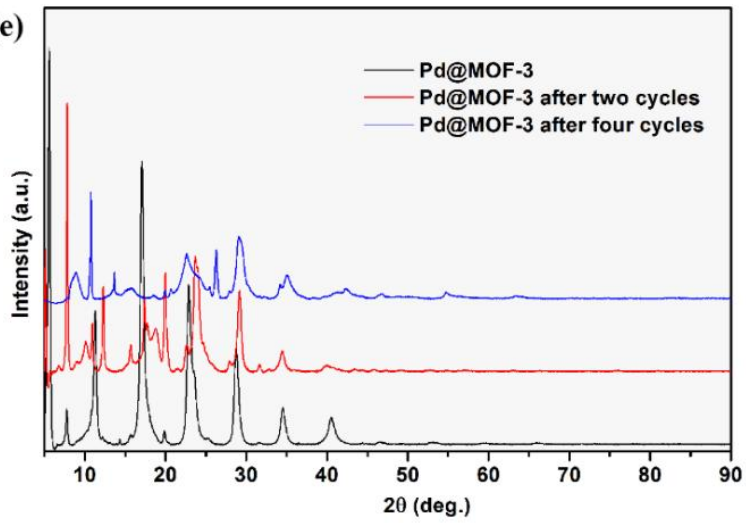

Figure S9. TEM images and PXRD of recovered Pd@MOF-3 catalyst after four Heck-coupling catalytic cycles. (a) Bright-field TEM image showing the Pd NPs are homogeneously dispersed in MOF-3. (b) Corresponding Pd NPs size distribution histogram. (c) Corresponding STEM bright field image. (d) EDX mapping of the Pd element into MOF-3. (e) PXRD pattern of Pd@MOF-3 after different cycles of Heck-coupling reaction. 

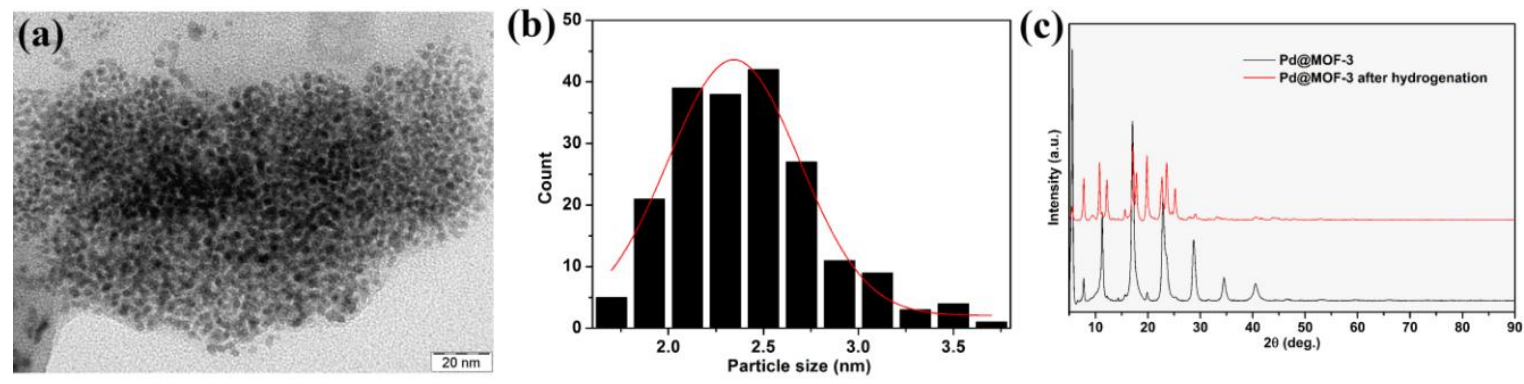

Figure S10. TEM image and PXRD of recovered Pd@MOF-3 catalyst after five hydrogenation catalytic cycles. (a) Bright-field TEM image showing the Pd NPs are homogeneously dispersed in MOF-3. (b) Corresponding Pd NPs size distribution histogram. (c) PXRD pattern of Pd@MOF-3 after different cycles of hydrogenation.
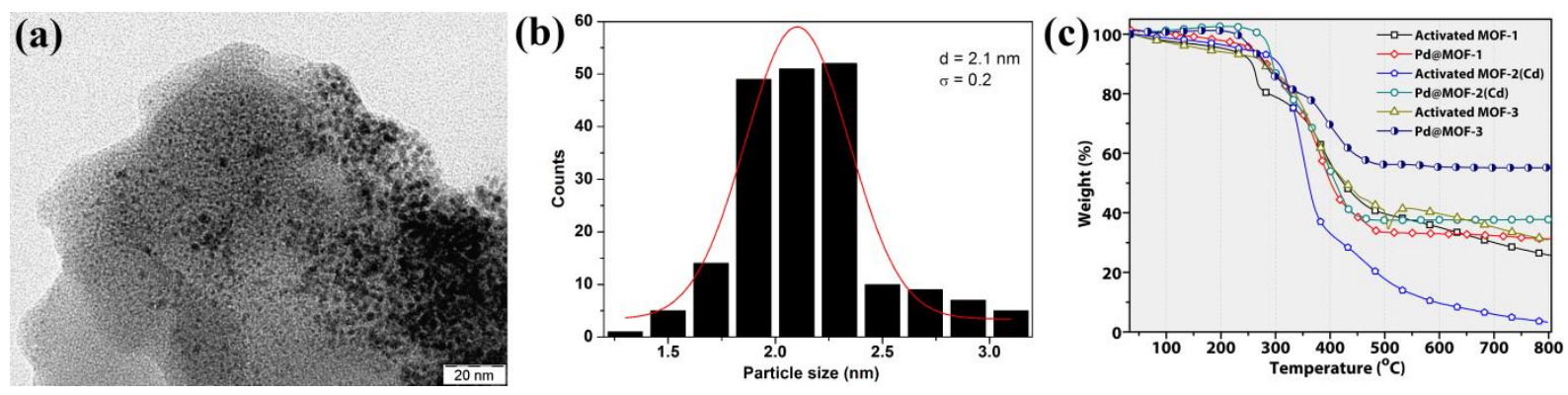

Figure S11: (a) Bright-field TEM image of the Pd@MOF-3 after annealing at $150{ }^{\circ} \mathrm{C}$ for $3 \mathrm{~h}$. (b) Corresponding particle size distribution. (c) TGA of Pd@MOFs comparing with their corresponding activated MOFs.

\section{Characterization of Heck-coupling reaction products:}

Methyl cinnamate (4a and 4d) (Table 1, entry 1 and 4). ${ }^{1} \mathrm{H}-\mathrm{NMR}\left(\mathrm{CDCl}_{3}\right) ; \delta(p p m): 7.719(\mathrm{~d}, 1 \mathrm{H}), 7.540-$

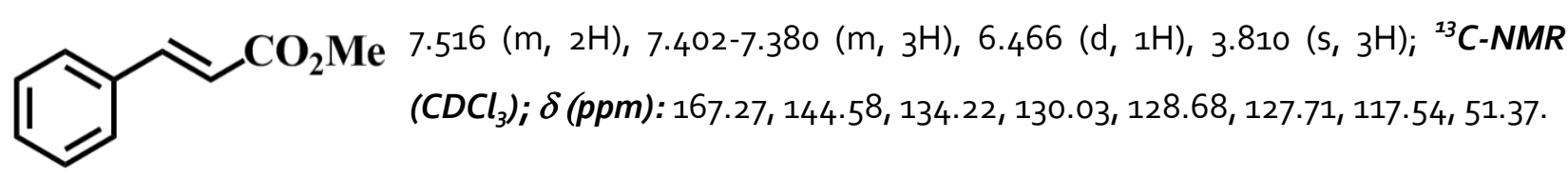

Ethyl cinnamate (4b) (Table 1, entry 2). $\left.{ }^{1} \mathrm{H}-\mathrm{NMR}(\mathrm{CDCl})_{3}\right) ; \delta(p p m): 7.709(\mathrm{~d}, 1 \mathrm{H}), 7.538-7.505(\mathrm{~m}, 2 \mathrm{H})$,

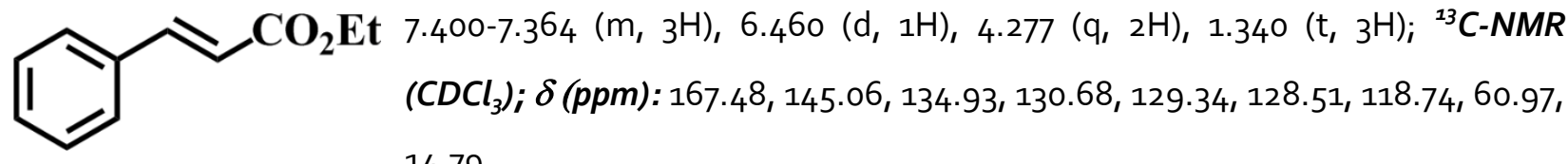
14.79 . 


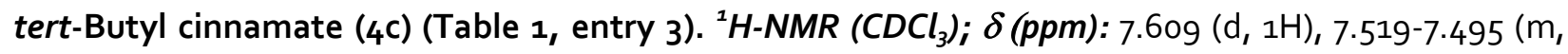
$\left.\mathrm{CO}_{2}{ }^{\mathrm{t}} \mathrm{Bu}^{2 \mathrm{H}}\right), 7.384-7.357(\mathrm{~m}, 3 \mathrm{H}), 6.391(\mathrm{~d}, 1 \mathrm{H}), 1.538(\mathrm{~s}, 9 \mathrm{H}){ }^{13}{ }^{13} \mathrm{C}-\mathrm{NMR}\left(\mathrm{CDCl}_{3}\right) ; \delta(p p m)$ :
$166.79,144.01,135.14,130.41,129.27,128.41,120.66,80.97,28.67$.

(E)-methyl 3-(p-tolyl)acrylate (4e and $4 \mathrm{f}$ ) (Table 1, entries 5 and 6). ${ }^{1} \mathrm{H}-\mathrm{NMR}\left(\mathrm{CDCl}_{3}\right) ; \delta(p p m): 7.682$

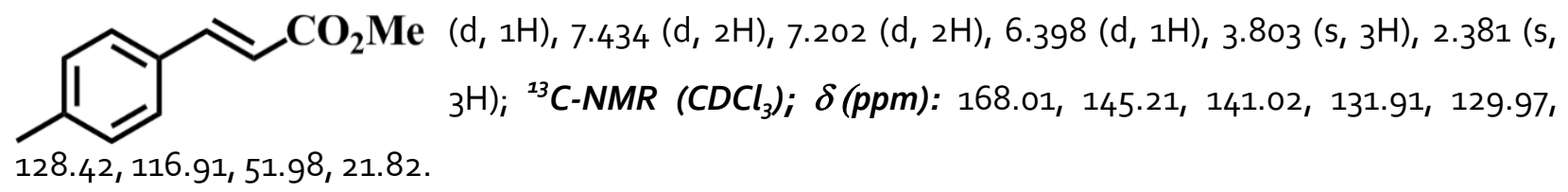<smiles>COC(=O)C=Cc1cc(C)cc(C)c1</smiles>

(E)-methyl 3-(3,5-dimethylphenyl)acrylate ( $4 \mathrm{~g}$ and $4 \mathrm{j})$ (Table 1, entries 7 and 10). ${ }^{1} H-N M R\left(C D C l_{3}\right) ; \delta(p p m): 7.660(\mathrm{~d}, 1 \mathrm{H}), 7.142(\mathrm{~s}, 2 \mathrm{H}), 7.025(\mathrm{~s}, 1 \mathrm{H})$, $6.433(\mathrm{~d}, 1 \mathrm{H}), 3.800(\mathrm{~s}, 3 \mathrm{H}), 2.329(\mathrm{~s}, 6 \mathrm{H}){ }^{13} \mathrm{C}-\mathrm{NMR}\left(\mathrm{CDCl}_{3}\right) ; \delta(p p m):$ 168.06, $145.69,138.88,134.76,132.57,126.42,117.79,52.11,21.65$.

(E)-ethyl 3-(3,5-dimethylphenyl)acrylate (4h) (Table 1, entry 8). ${ }^{1} \mathrm{H}-N M R\left(C D C l_{3}\right) ; \delta(p p m): 7.650(\mathrm{~d}$, $\left.\mathbf{C O}_{2} \mathbf{E t}{ }_{1 \mathrm{H}}\right), 7.145(\mathrm{~s}, 2 \mathrm{H}), 7.020(\mathrm{~s}, 1 \mathrm{H}), 6.431(\mathrm{~d}, 1 \mathrm{H}), 4.266(\mathrm{q}, 2 \mathrm{H}), 2.327(\mathrm{~s}, 6 \mathrm{H})$,
$1.335\left(\mathrm{t},{ }_{3} \mathrm{H}\right) ;{ }^{13} \mathrm{C}-N M R\left(C D C l_{3}\right) ; \delta(p p m): 167.62,145.38,138.85,134.85$,
$132.48,126.40,118.29,60.87,21.65,14.79$.

(E)-tert-butyl 3-(3,5-dimethylphenyl)acrylate (4i) (Table 1, entry 9). ${ }^{1} \mathrm{H}-\mathrm{NMR}\left(\mathrm{CDCl}_{3}\right) ; \delta(p p m): 7.548$ ${ }^{13} \mathrm{C}-\mathrm{NMR}\left(\mathrm{CDCl}_{3}\right) ; \delta(p p m): 166.95,144.32,138.78,135.05,132.21,126.30$,
$120.22,80.81,28.68,21.65$. (E)-methyl 3-(4-methoxyphenyl)acrylate (4k and $4 \mathrm{l}$ ) (Table 1, entries 11 and ${ }^{12}$ ). ${ }^{1} \mathrm{H}-\mathrm{NMR}\left(\mathrm{CDCl}_{3}\right)$; $\delta(p p m): 7.661(\mathrm{~d}, 1 \mathrm{H}), 7.483(\mathrm{~d}, 2 \mathrm{H}), 6.908(\mathrm{~d}, 2 \mathrm{H}), 6.325(\mathrm{~d}, 1 \mathrm{H}), 3.843(\mathrm{~s}$,
$\begin{aligned} & 3 \mathrm{H}), 3.801(\mathrm{~s}, 3 \mathrm{H}) ;{ }^{13} \mathrm{C}-\mathrm{NMR}\left(\mathrm{CDCl}_{3}\right) ; \delta(p p m): 168.01,161.68,144.81 \text {, } \\ & 130.02,127.28,115.51,114.57,55.68,51.87 .\end{aligned}$

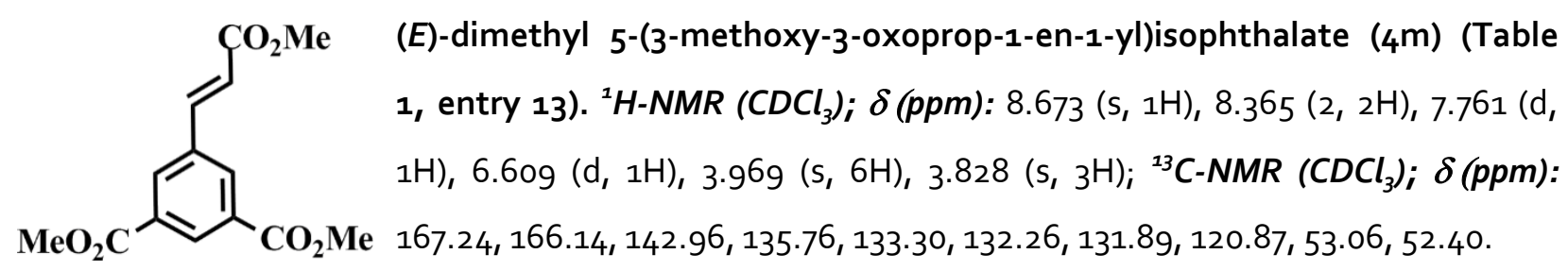


(E)-1,2-diphenylethene (6a and 6b) (Table 2, entries 1 and 2). ${ }^{1} \mathrm{H}-\mathrm{NMR}\left(\mathrm{CDCl}_{3}\right) ; \delta(p p m): 7.545(\mathrm{~d}, 4 \mathrm{H})$,

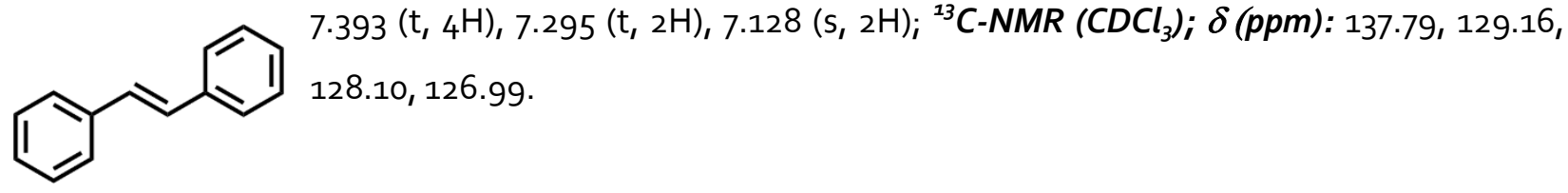<smiles>Cc1ccc(/C=C/c2ccccc2)cc1</smiles>

(E)-1-methyl-4-styrylbenzene (6c and 6d) (Table 2, entries 3 and 4 ). ${ }^{1} H$-NMR $\left(C D C l_{3}\right) ; \delta(p p m): 7.532(\mathrm{~d}, 2 \mathrm{H}), 7.443(\mathrm{~d}, 2 \mathrm{H}), 7.385(\mathrm{t}, 2 \mathrm{H}), 7.280(\mathrm{t}, 1 \mathrm{H}), 7.195$ $(\mathrm{d}, 2 \mathrm{H}), 7.096(\mathrm{~s}, 2 \mathrm{H}), 2.377(\mathrm{~s}, 3 \mathrm{H}) ;{ }^{13} \mathrm{C}-\mathrm{NMR}\left(\mathrm{CDCl}_{3}\right) ; \delta(p p m): 137.99,135.02$, $129.87,129.13,129.09,128.17,127.88,126.90,126.87,21.74$.

(E)-1,3-dimethyl-5-styrylbenzene (6e and 6f) (Table 2, entries 5 and 6). ${ }^{1} \mathrm{H}-\mathrm{NMR}\left(\mathrm{CDCl}_{3}\right) ; \delta(p p m)$ :<smiles>Cc1cc(C)cc(/C=C/c2ccccc2)c1</smiles>
$7.547(\mathrm{~d}, 2 \mathrm{H}), 7.402(\mathrm{t}, 2 \mathrm{H}), 7.299(\mathrm{t}, 1 \mathrm{H}), 7.178(\mathrm{~s}, 2 \mathrm{H}), 7.108(\mathrm{~d}, 2 \mathrm{H}), 6.944(\mathrm{~s}$, $1 \mathrm{H}), 2.371(\mathrm{~s}, 6 \mathrm{H}) ;{ }^{13} \mathrm{C}-\mathrm{NMR}\left(\mathrm{CDCl}_{3}\right) ; \delta(\mathrm{ppm}): 138.59,137.99,137.69,129.91$, $129.35,129.13,128.76,127.94,126.92,124.91,21.79$.

(E)-1-methoxy-4-styrylbenzene (6g and 6h) (Table 2, entries 7 and 8). ${ }^{1} \mathrm{H}-\mathrm{NMR}\left(\mathrm{CDCl}_{3}\right) ; \delta(\mathrm{ppm}): 7.361$ $(\mathrm{d}, 2 \mathrm{H}), 7.329(\mathrm{~d}, 2 \mathrm{H}), 7.262(\mathrm{t}, 2 \mathrm{H}), 7.128(\mathrm{t}, 1 \mathrm{H}), 6.941(\mathrm{~d}, 1 \mathrm{H}), 6.845(\mathrm{~d}, 1 \mathrm{H}), 6.763(\mathrm{~d}, 2 \mathrm{H}), 3.711(\mathrm{~s}, 3 \mathrm{H})$;

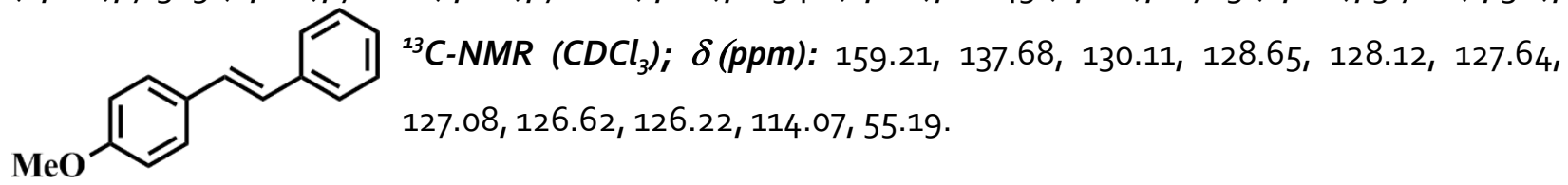
(E)-methyl 3-(4-bromophenyl)acrylate (9a) (Table 3, entry 1). ${ }^{1} \mathrm{H}-\mathrm{NMR}\left(\mathrm{CDCl}_{3}\right) ; \delta(\mathrm{ppm}): 7.638(\mathrm{~d}, \mathrm{1H})$,

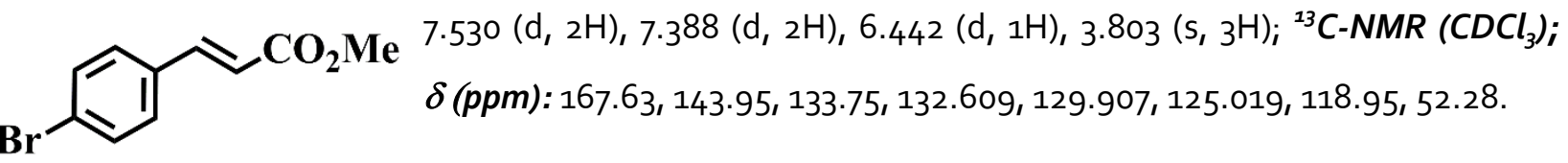
(E)-1-bromo-4-styrylbenzene (gb) (Table 3, entry 3). ${ }^{1} \mathrm{H}-\mathrm{NMR}\left(\mathrm{CDCl}_{3}\right) ; \delta(p p m): 7.518(\mathrm{~m}, 4 \mathrm{H}), 7.391$ $(\mathrm{m}, 4 \mathrm{H}), 7.297(\mathrm{t}, 1 \mathrm{H}), 7.083(\mathrm{~d}, 1 \mathrm{H}), 7.054(\mathrm{~d}, 1 \mathrm{H}) ;{ }^{13} C-N M R\left(C D C l_{3}\right) ; \delta(p p m)$ :
$137.41,136.74,132.25,129.88,129.22,128.44,128.38,127.86,127.03,121.78$. (2E,2E')-dimethyl-3,3'-(1,4-phenylene)diacrylate (10a) (Table 3, entry 2). ${ }^{1} \mathrm{H}-\mathrm{NMR}\left(\mathrm{CDCl}_{3}\right) ; \delta(p p m)$ :

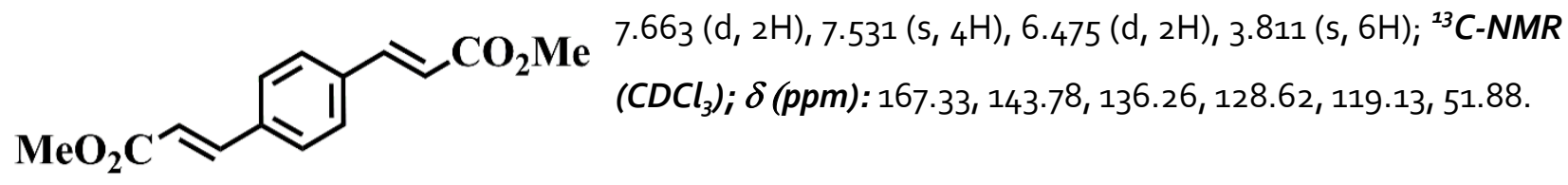


$(E, E)$-1,4-distyrylbenzene (1ob) (table 3, entry 4). ${ }^{1} \mathrm{H}-\mathrm{NMR}\left(\mathrm{CDCl}_{3}\right) ; \delta(\mathrm{ppm}): 7.542-7.508(\mathrm{~m}, 8 \mathrm{H})$,<smiles>C(=C/c1ccc(/C=C/c2ccccc2)cc1)\c1ccccc1</smiles>
7.392-7.351 (m, 4H), 7.282-7.253 (m, 2H), $7.121(\mathrm{~s}, 4 \mathrm{H}) ;{ }^{13} \mathrm{C}-N M R$ $\left(\mathrm{CDCl}_{3}\right) ; \delta(\mathrm{ppm}): 137.31,136.74,128.68,128.62,128.31,127.73,126.91$, 126.49 .

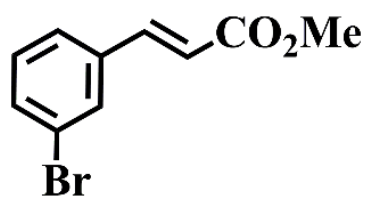

(E)-methyl 3-(3-bromophenyl)acrylate (12a) (Table 4, entry 1). ${ }^{1} H$-NMR $\left(C D C l_{3}\right) ; \delta(p p m): 7.688(s, 1 H), 7.679(\mathrm{~d}, 1 \mathrm{H}), 7.538(\mathrm{~d}, 1 \mathrm{H}), 7.465(\mathrm{~d}, 1 \mathrm{H}), 7.299$ $(\mathrm{t}, 1 \mathrm{H}), 6.472(\mathrm{~d}, 1 \mathrm{H}), 3.83(\mathrm{~s}, 3 \mathrm{H}) ;{ }^{13} \mathrm{C}-N M R\left(\mathrm{CDCl}_{3}\right) ; \delta(p p m): 167.44,143.60$, $136.93,133.53,131.21,130.86,127.14,123.49,119.75,52.32$.

(E)-1-bromo-3-styrylbenzene (12b) (Table 4, entry 3). ${ }^{1} \mathrm{H}-N M R\left(C D C l_{3}\right) ; \delta(p p m): 7.678(\mathrm{~s}, 1 \mathrm{H}), 7.522(\mathrm{~d}$, $2 \mathrm{H}), 7.431(\mathrm{~m}, 4 \mathrm{H}), 7.311(\mathrm{~m}, 2 \mathrm{H}), 7.092(\mathrm{~d}, 1 \mathrm{H}), 7.043(\mathrm{~d}, 1 \mathrm{H}) ;{ }^{13} \mathrm{C}-N M R\left(C D C l_{3}\right) ;$
$\delta(p p m): 139.99,137.25,130.86,130.63,129.70,129.23,128.53,127.56,127.13$, (2E,2 $\left.E^{\prime}\right)$-dimethyl-3,3'-(1,3-phenylene)diacrylate (13a) (Table 4, entry 2). ${ }^{1} \mathrm{H}-\mathrm{NMR}\left(\mathrm{CDCl}_{3}\right) ; \delta(p p m)$ : $6 \mathrm{H}) ;{ }^{13} \mathrm{C}-\mathrm{NMR}(\mathrm{CDCl}) ; \delta(\mathrm{ppm}): 168.82,145.47,137.73,137.71,131.14,129.36$,
$120.42,53.48$. (E,E)-1,3-Distyrylbenzene (13b) (Table 4, entry 4). ${ }^{1} \mathrm{H}-\mathrm{NMR}\left(\mathrm{CDCl}_{3}\right) ; \delta(p p m): 7.583(\mathrm{~s}, 1 \mathrm{H}), 7.467(\mathrm{~d}$,<smiles>C(=C/c1cccc(/C=C/c2ccccc2)c1)\c1ccccc1</smiles>
$4 \mathrm{H}), 7.373-7.291(\mathrm{~m}, 7 \mathrm{H}), 7.231-7.168(\mathrm{~m}, 2 \mathrm{H}), 7.122-7.041(\mathrm{~m}, 4 \mathrm{H}) ;{ }^{13} \mathrm{C}$ NMR $\left(\mathrm{CDCl}_{3}\right) ; \delta(\mathrm{ppm}): 137.34,137.21,129.05,128.73,128.55,127.76$, $126.51,125.68,124.69$. 


\section{Hydrogenation Products:}

Ethyl benzene (18a): ${ }^{1} \mathrm{H}-\mathrm{NMR}\left(\mathrm{CDCl}_{3}\right) ; \delta(\mathrm{ppm}): 7.52-7.38(\mathrm{~m}, 2 \mathrm{H}), 7.33-7.16(\mathrm{~m}, 3 \mathrm{H}) 2.65(\mathrm{q}, 2 \mathrm{H}), 1.24(\mathrm{t}$, $3 \mathrm{H}) i^{13} \mathrm{C}-\mathrm{NMR}\left(\mathrm{CDCl}_{3}\right) ; \delta(\mathrm{ppm}): 144.25,128.28,127.84,125.57,28.87,15.68$.

1-ethyl-4-methylbenzene (18b): ${ }^{1} H$ NMR $\left(\mathrm{CDCl}_{3}\right) ; \delta(\mathrm{ppm}): 7.10(\mathrm{~s}, 4 \mathrm{H}), 2.61$ (q,<smiles>CCc1ccc(C)cc1</smiles>
$2 \mathrm{H}), 2.32(\mathrm{~s}, 3 \mathrm{H}), 1.23(\mathrm{t}, 3 \mathrm{H}) ;{ }^{13} \mathrm{C}-\mathrm{NMR}\left(\mathrm{CDCl}_{3}\right) ; \delta(p p m): 141.19,134.95,128.97$, $127.71,28.43,20.96,15.75$.

1-Chloro-4-ethylbenzene (18c): ${ }^{1} \mathrm{H} N M R\left(\mathrm{CDCl}_{3}\right) ; \delta(p p m): 7.23(\mathrm{~d}, 2 \mathrm{H}), 7.12(\mathrm{~d}, 2 \mathrm{H})$,<smiles>CCc1ccc(Cl)cc1</smiles>
1,2-Diphenylethane (18d): ${ }^{1} \mathrm{H} N M R\left(\mathrm{CDCl}_{3}\right) ; \delta(\mathrm{ppm}): 7.31-7.26(\mathrm{~m}, 4 \mathrm{H}), 7.21-$<smiles>c1ccc(CCc2ccccc2)cc1</smiles>
$7.15(\mathrm{~m}, 6 \mathrm{H}), 2.91(\mathrm{~s}, 4 \mathrm{H}) ;{ }^{13} \mathrm{C}-\mathrm{NMR}\left(\mathrm{CDCl}_{3}\right) ; \delta(\mathrm{ppm}): 141.72,128.42,128.31$, $125.91,37.93$.

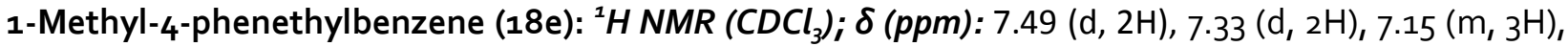<smiles>Cc1ccc(CCc2ccc([18OH])cc2)cc1</smiles>

1,3-Dimethyl-5-phenethylbenzene (18f): ${ }^{1} \mathrm{H} N M R\left(\mathrm{CDCl}_{3}\right) ; \delta(p p m): 7.32(\mathrm{~m}, 5 \mathrm{H}), 6.86(\mathrm{~s}, 3 \mathrm{H}), 2.91(\mathrm{~d}$,<smiles>Cc1cc(C)cc(CCc2ccccc2)c1</smiles>
$4 \mathrm{H}), 2.31(\mathrm{~s}, 6 \mathrm{H}) ;{ }^{13} \mathrm{C}-\mathrm{NMR}\left(\mathrm{CDCl}_{3}\right) ; \delta(\mathrm{ppm}): 142.54,142.27,138.29,128.87$, $128.80,128.02,126.71,126.34,38.57,38.38,21.32$.

1-bromo-4-phenethylbenzene (18g): ${ }^{1} H$ NMR $\left(\mathrm{CDCl}_{3}\right) ; \delta(p p m)$ : 7.37 (d, 2H), 7.29-7.12 (m, 5H), $7.01(\mathrm{~d}$,<smiles>Brc1ccc(CCc2ccccc2)cc1</smiles>
$2 \mathrm{H}), 2.88(\mathrm{~s}, 4 \mathrm{H}) ;{ }^{13} \mathrm{C}-\mathrm{NMR}\left(\mathrm{CDCl}_{3}\right) ; \delta(\mathrm{ppm}): 141.21,140.58,131.30,130.22$, $128.39,128.32,126.02,119.57,37.73,37.2$. 
Methyl 3-phenylpropanoate (18h): ${ }^{1} H$ NMR $\left(\mathrm{CDCl}_{3}\right) ; \delta(p p m)$ : 7.31-7.28 (m, 2H), 7.22-7.19 (m, 3H), 3.68<smiles>COC(=O)CCc1ccccc1</smiles>

Methyl 3-(3,5-dimethylphenyl)propanoate (18i): ${ }^{1} \mathrm{H} N M R\left(\mathrm{CDCl}_{3}\right) ; \delta(p p m): 6.85(\mathrm{~s}, 1 \mathrm{H}), 6.82(\mathrm{~s}, 2 \mathrm{H})$,<smiles>COC(=O)CCc1cc(C)cc(C)c1</smiles>

Methyl 3-(4-bromophenyl)propanoate (18j): ${ }^{1} H$ NMR $\left(C C_{3}\right) ; \delta(p p m): 7.91(\mathrm{~d}, 2 \mathrm{H}), 7.17(\mathrm{~d}, 2 \mathrm{H}), 3.68(\mathrm{~s}$,<smiles>COC(=O)CCc1ccc(Br)cc1</smiles>

Dimethyl 5-(3-methoxy-3-oxopropyl)isophthalate (18k): ${ }^{1} H$ NMR $\left(\mathrm{CDCl}_{3}\right) ; \delta(p p m): 8.53(\mathrm{~s}, 1 \mathrm{H}), 8.08$

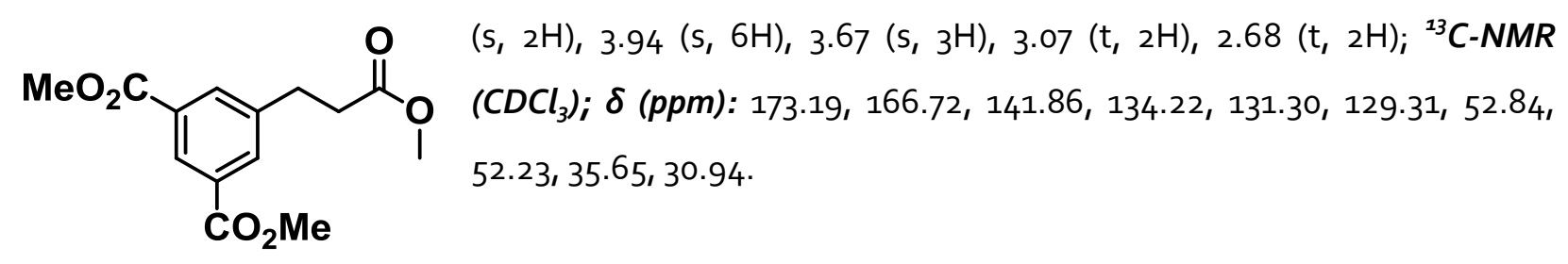



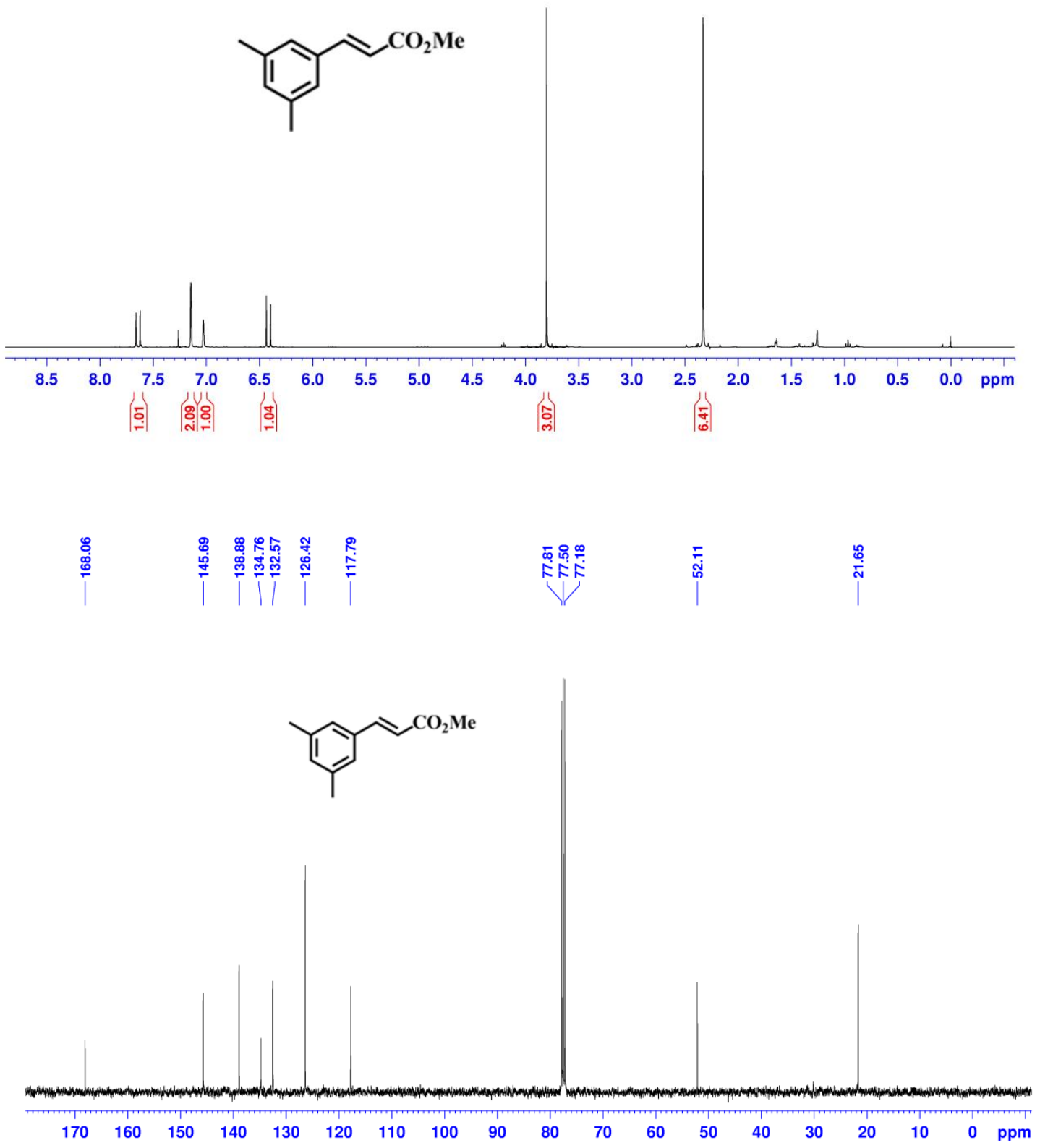


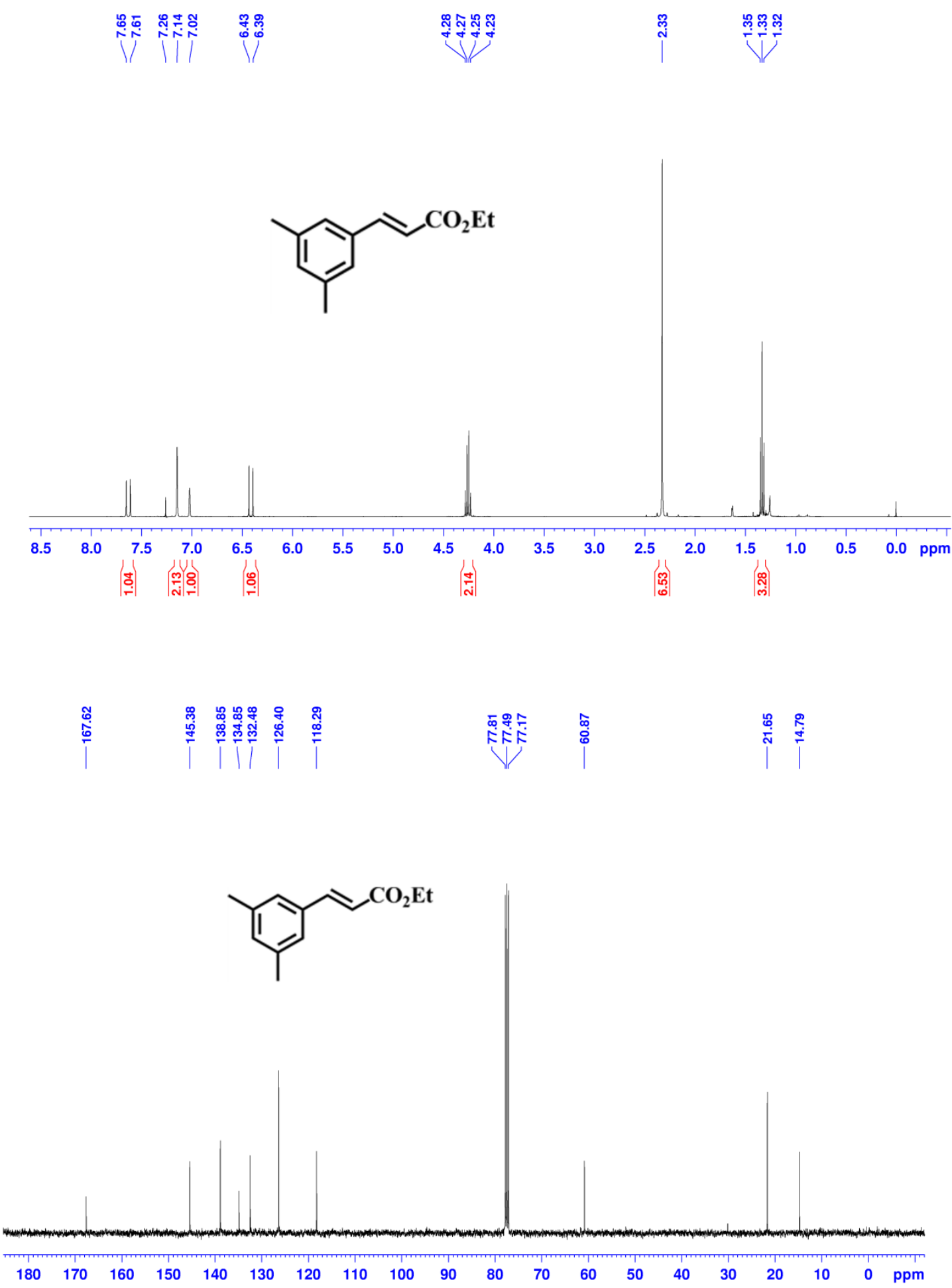




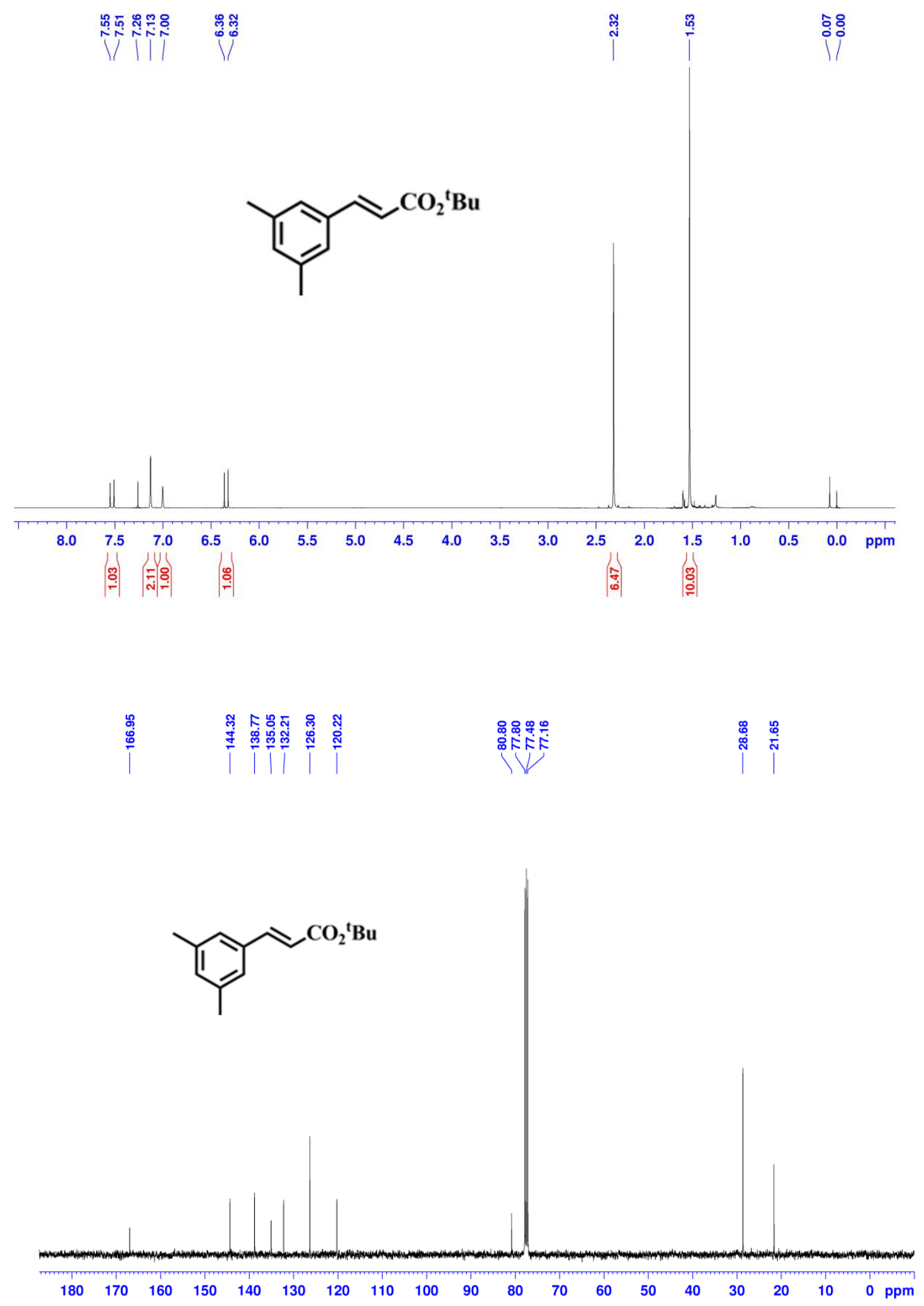



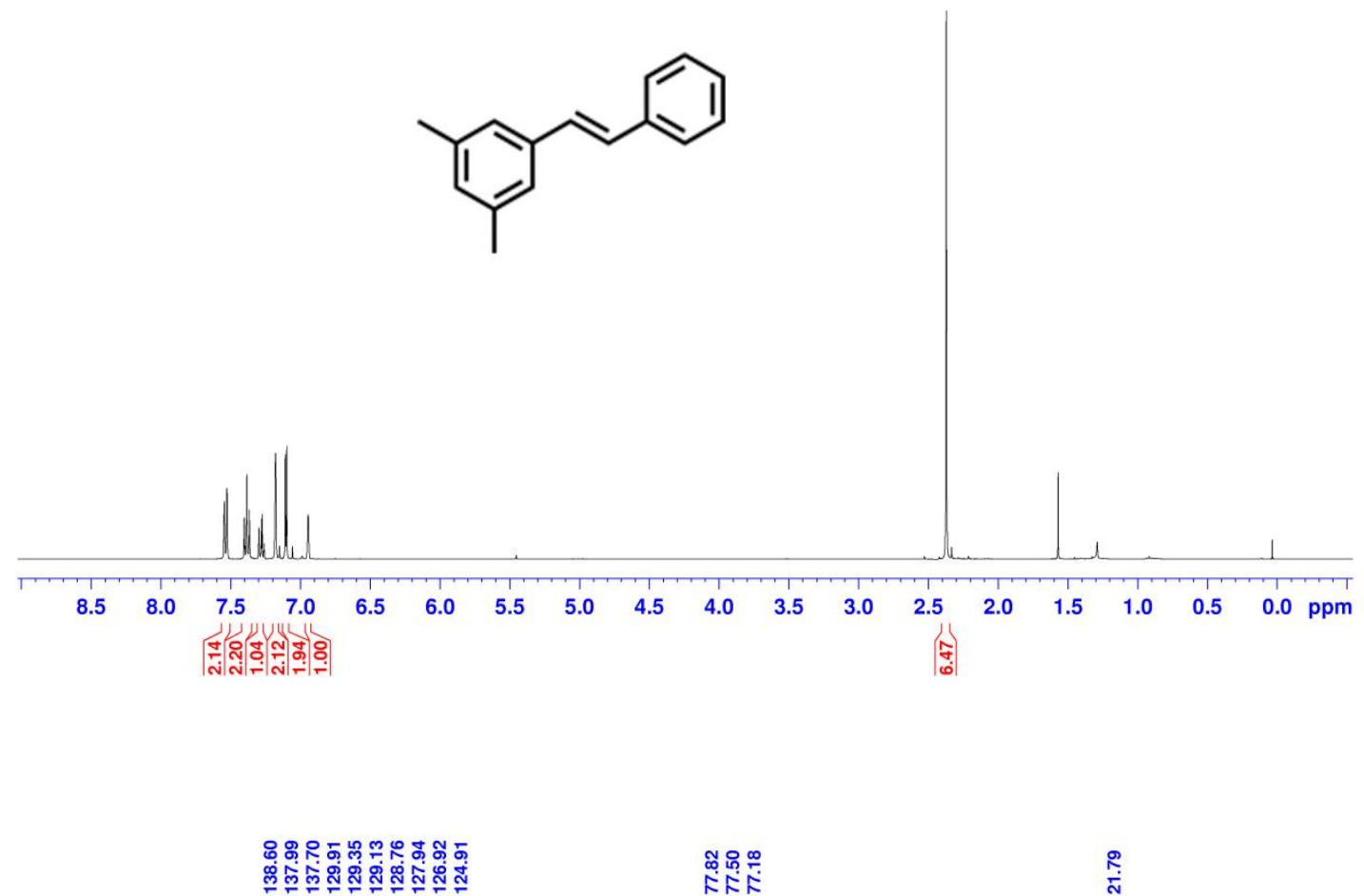

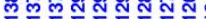

赵

Vर

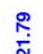

1 V/ll

กิ<smiles>Cc1cc(C)cc(/C=C/c2ccccc2)c1</smiles>

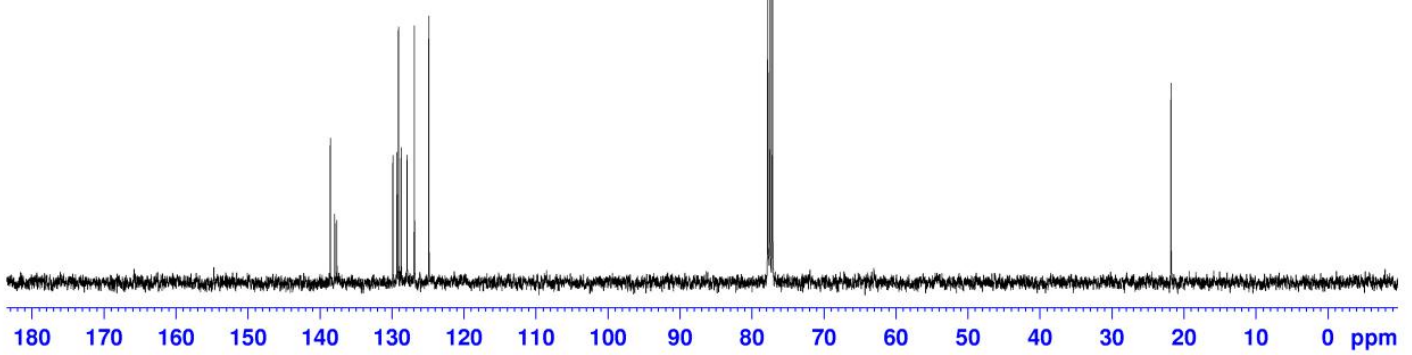




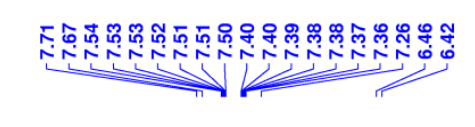

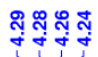

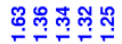

$\sqrt{1}$<smiles>CCOC(=O)C=Cc1ccccc1</smiles>
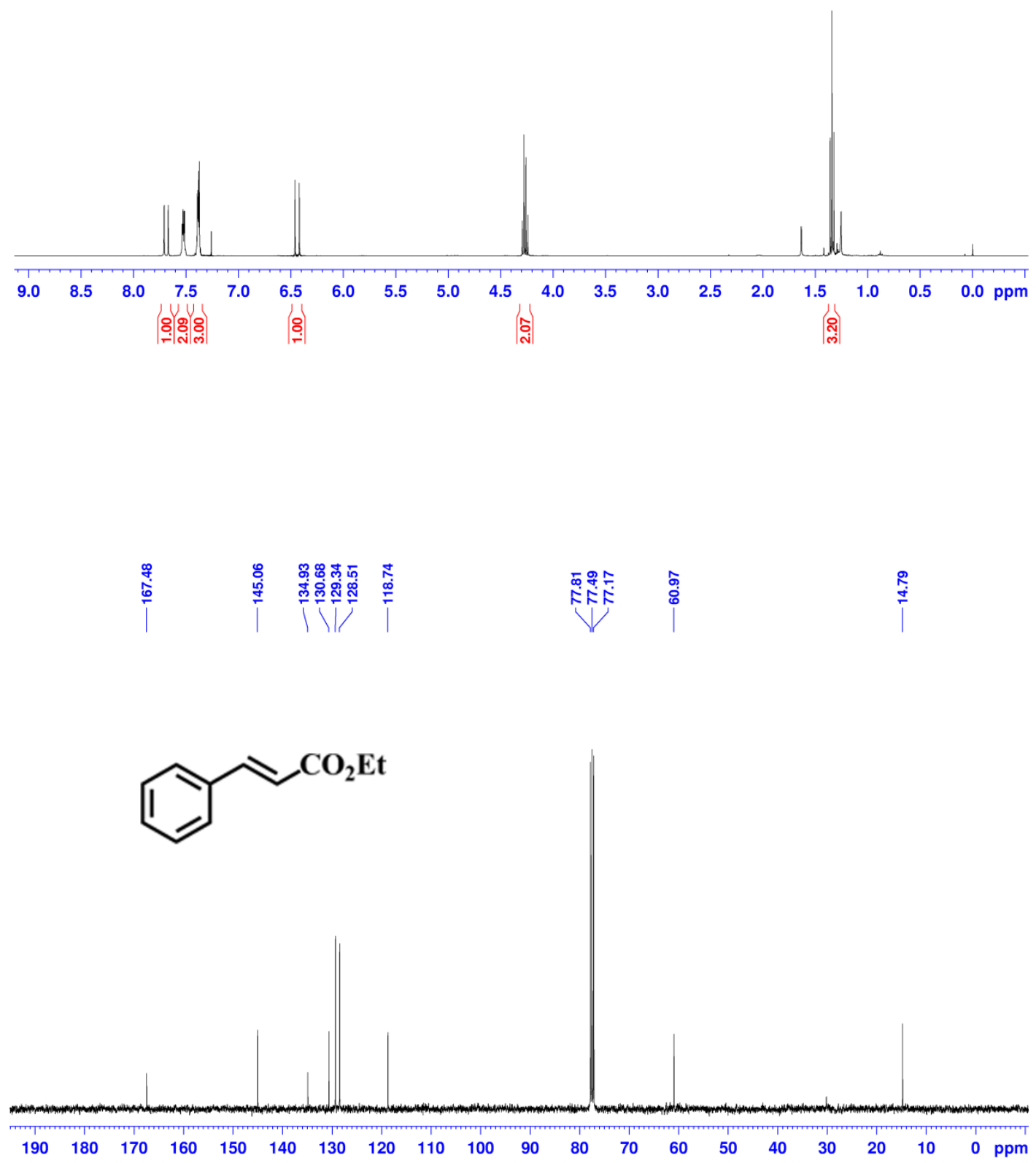


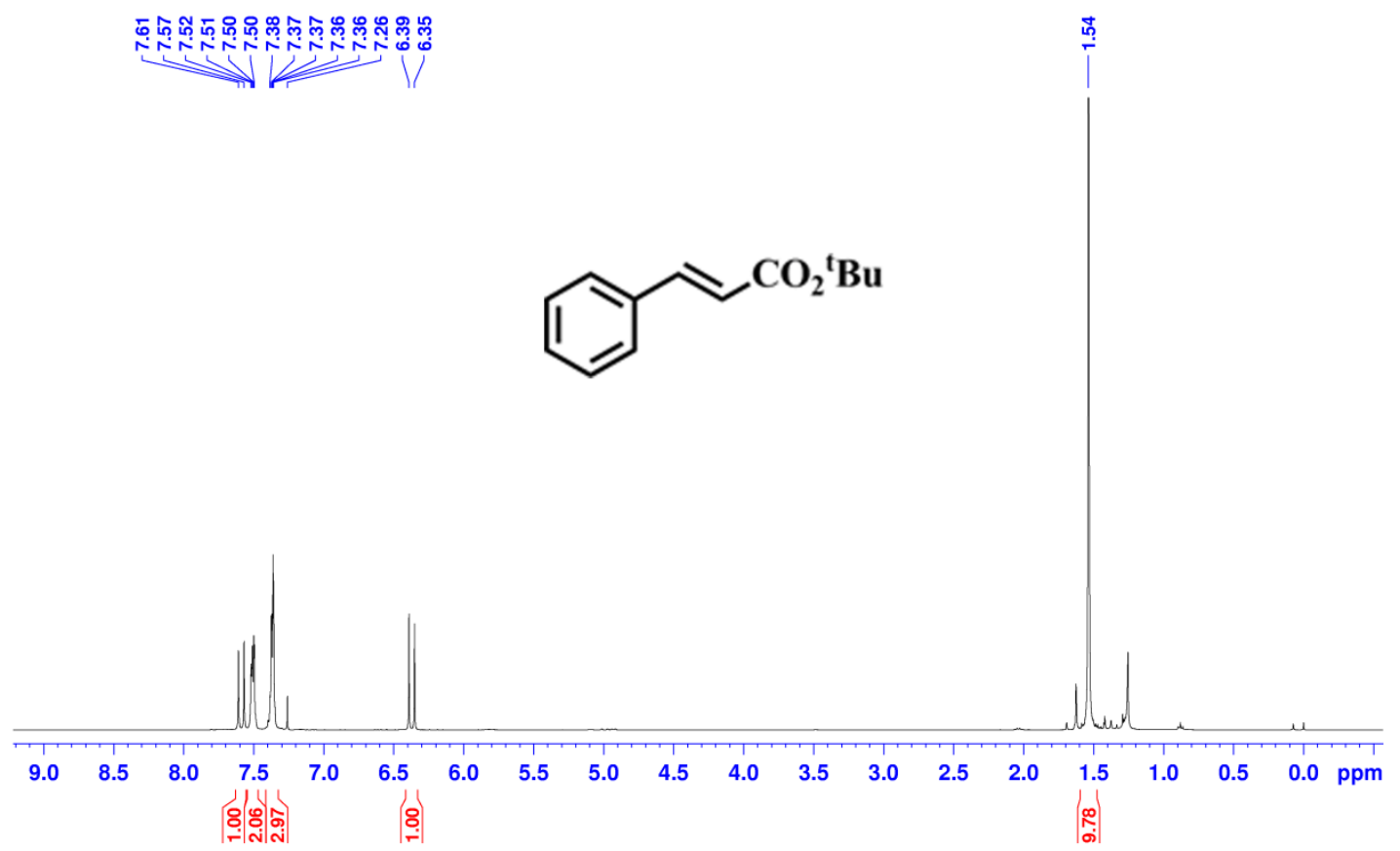

|

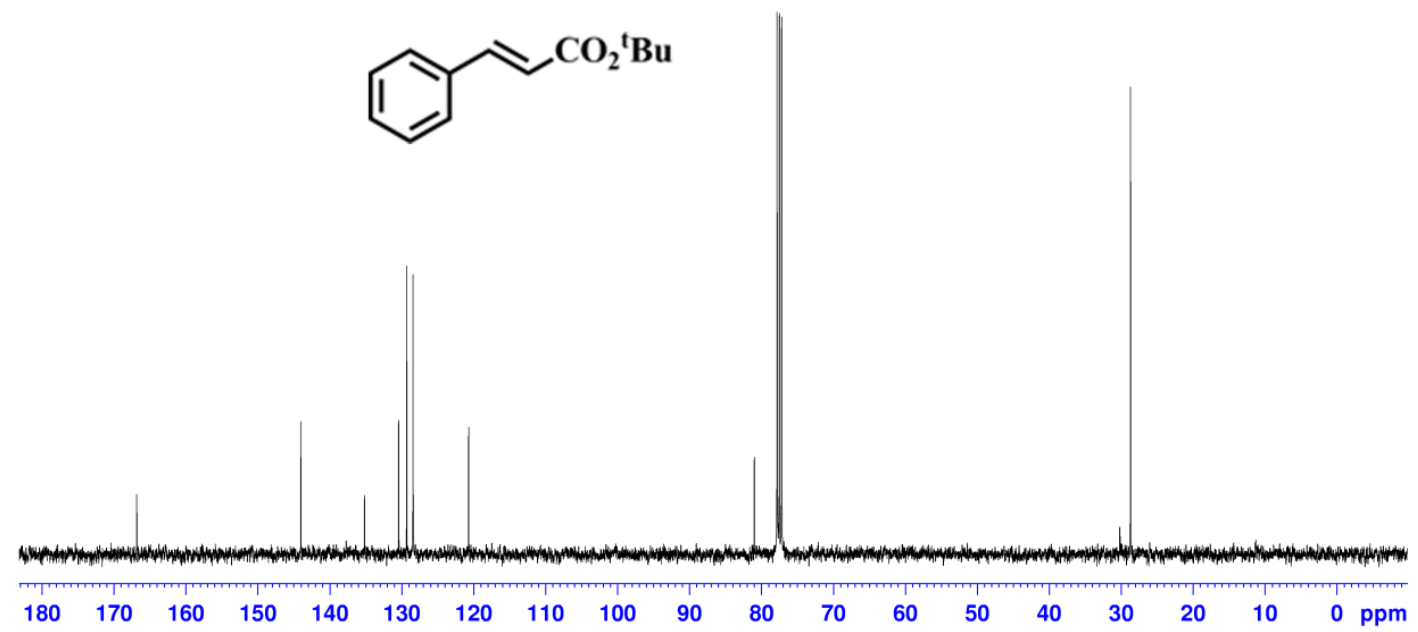




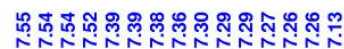
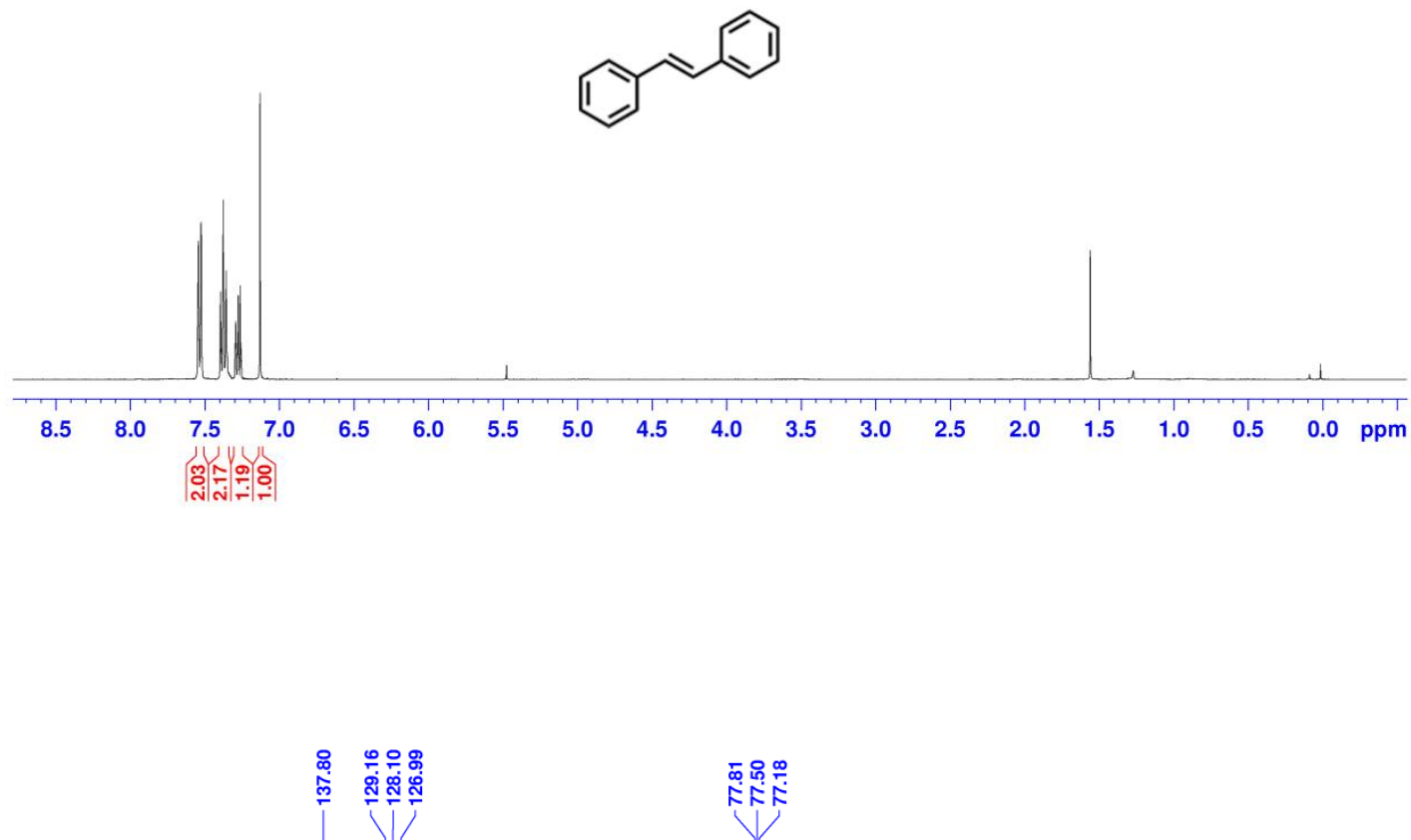

承虽尔

VरF

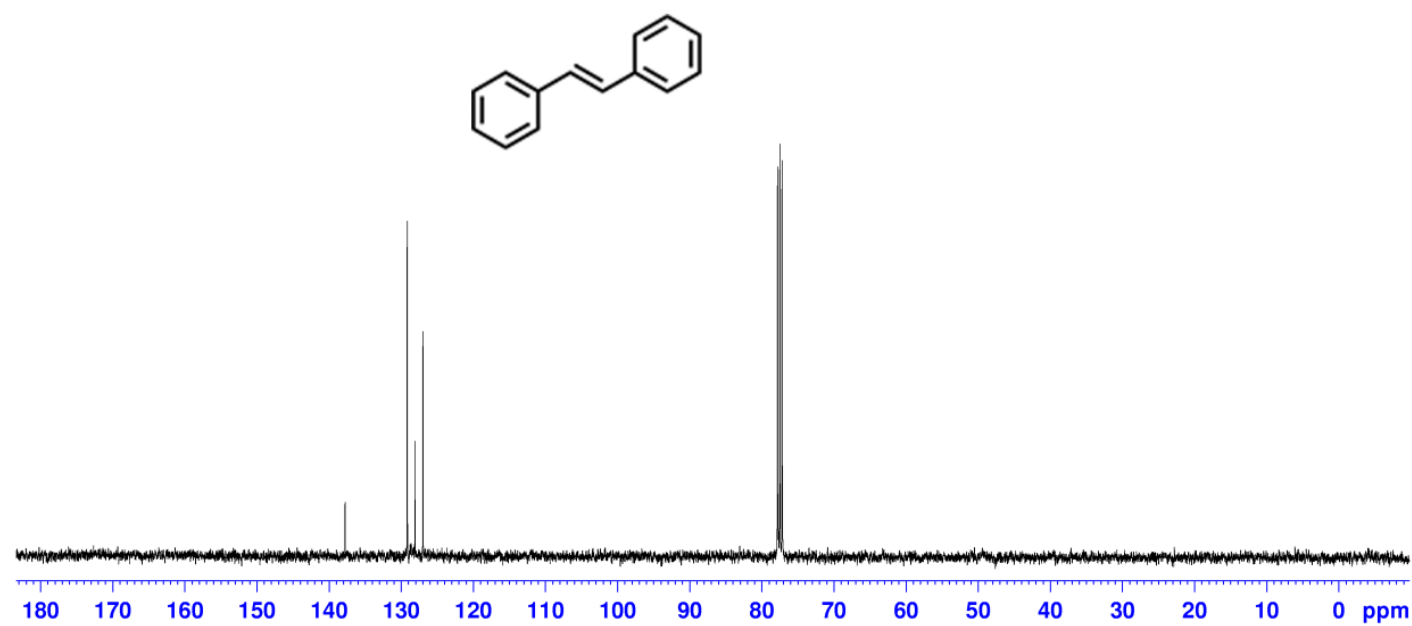



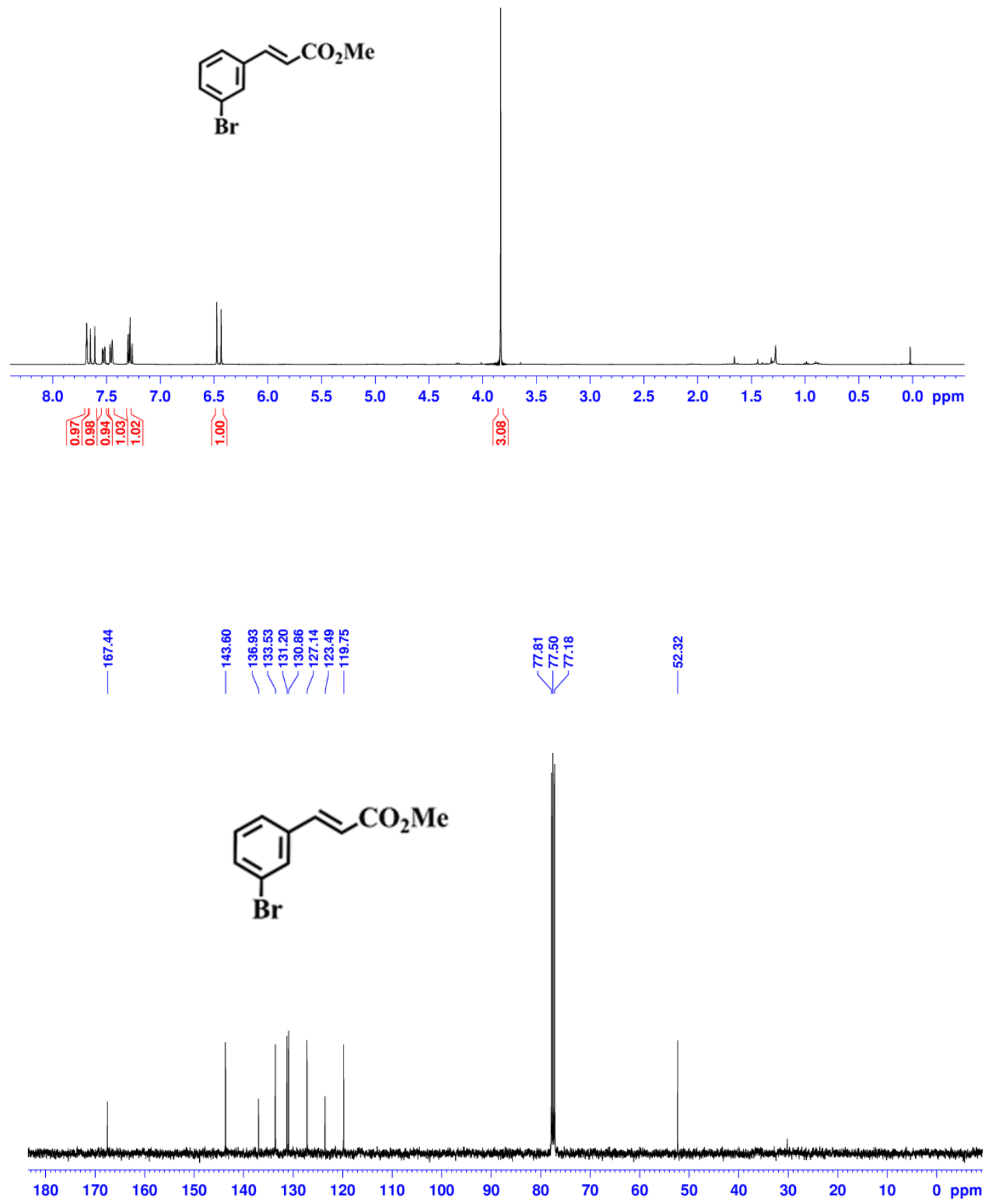


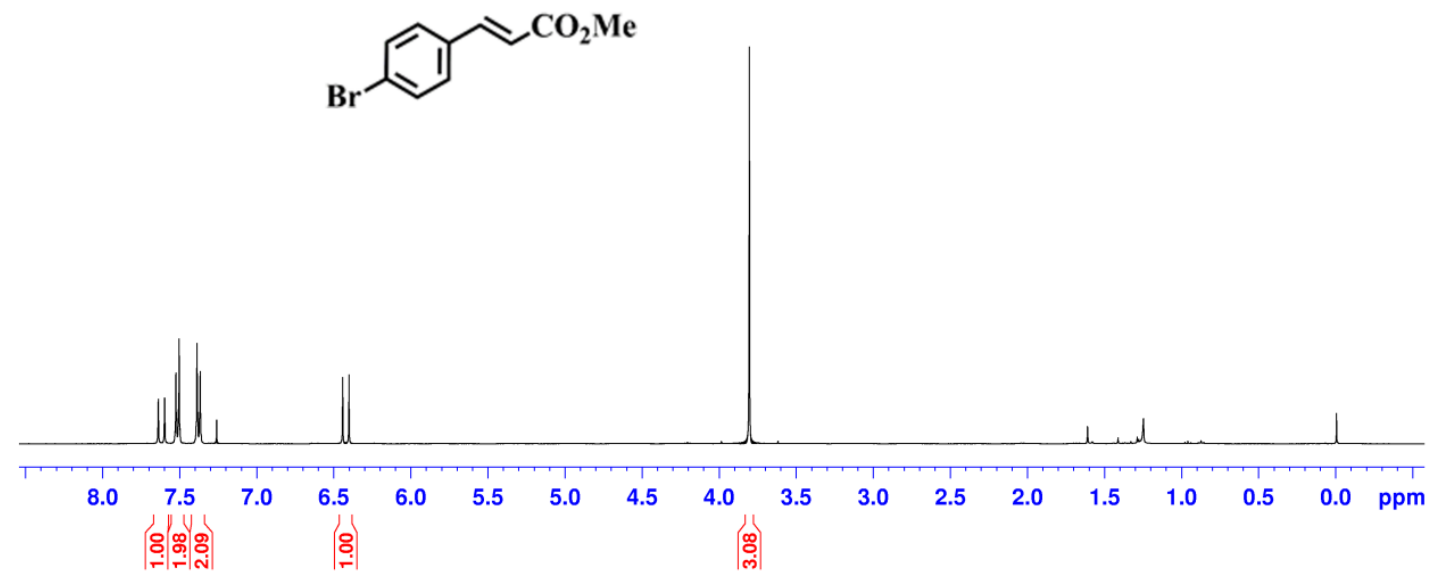

|

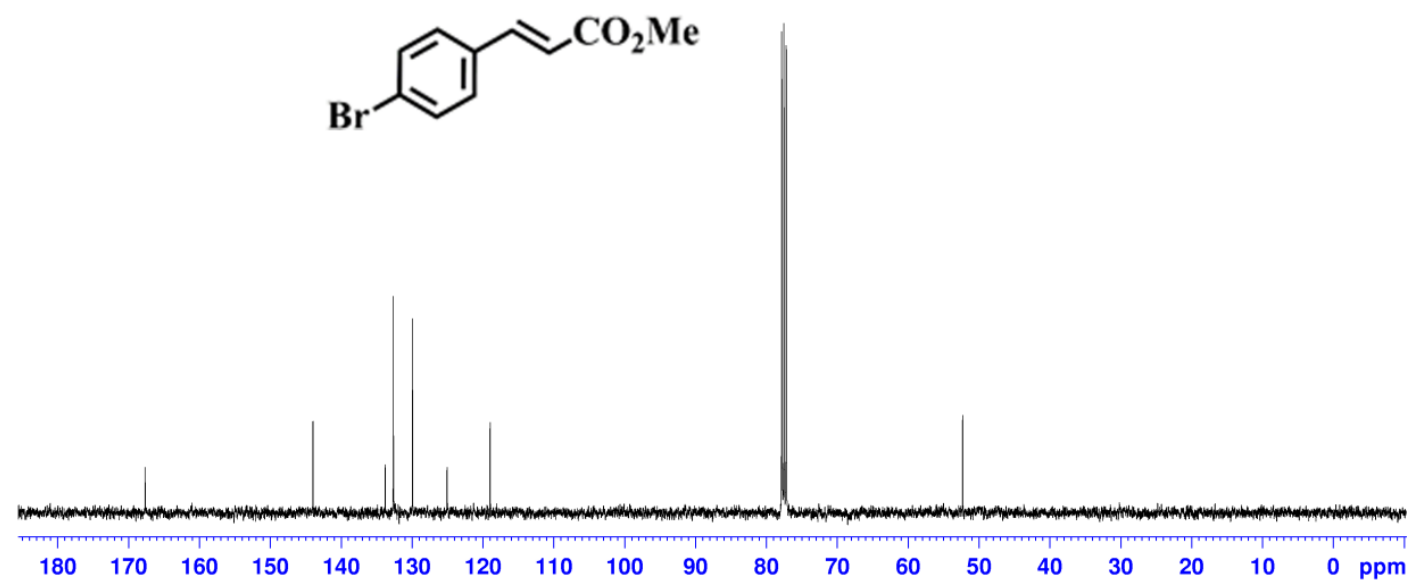




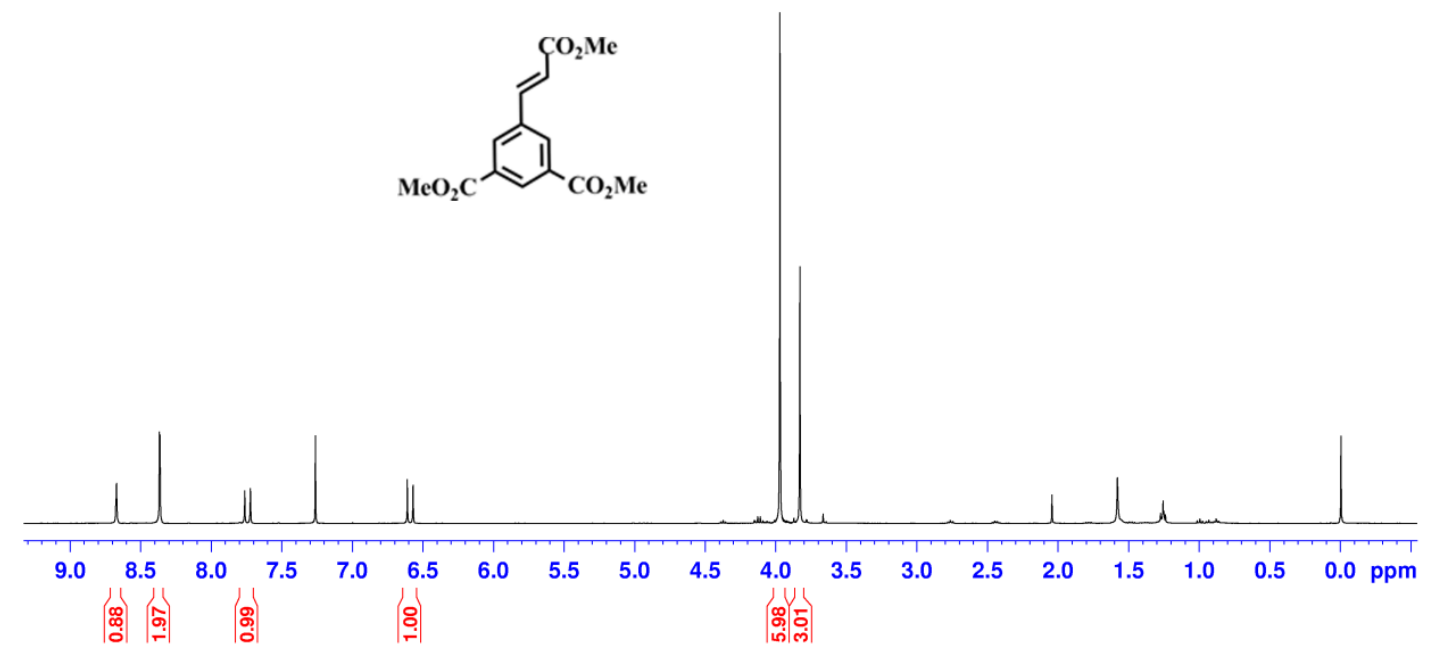

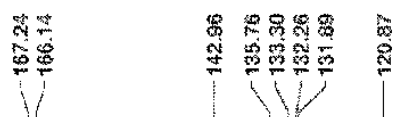
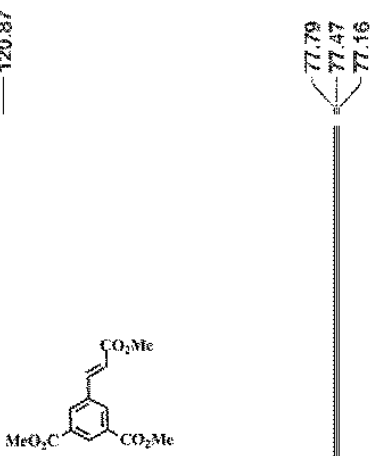

옹요

)

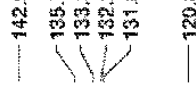

E

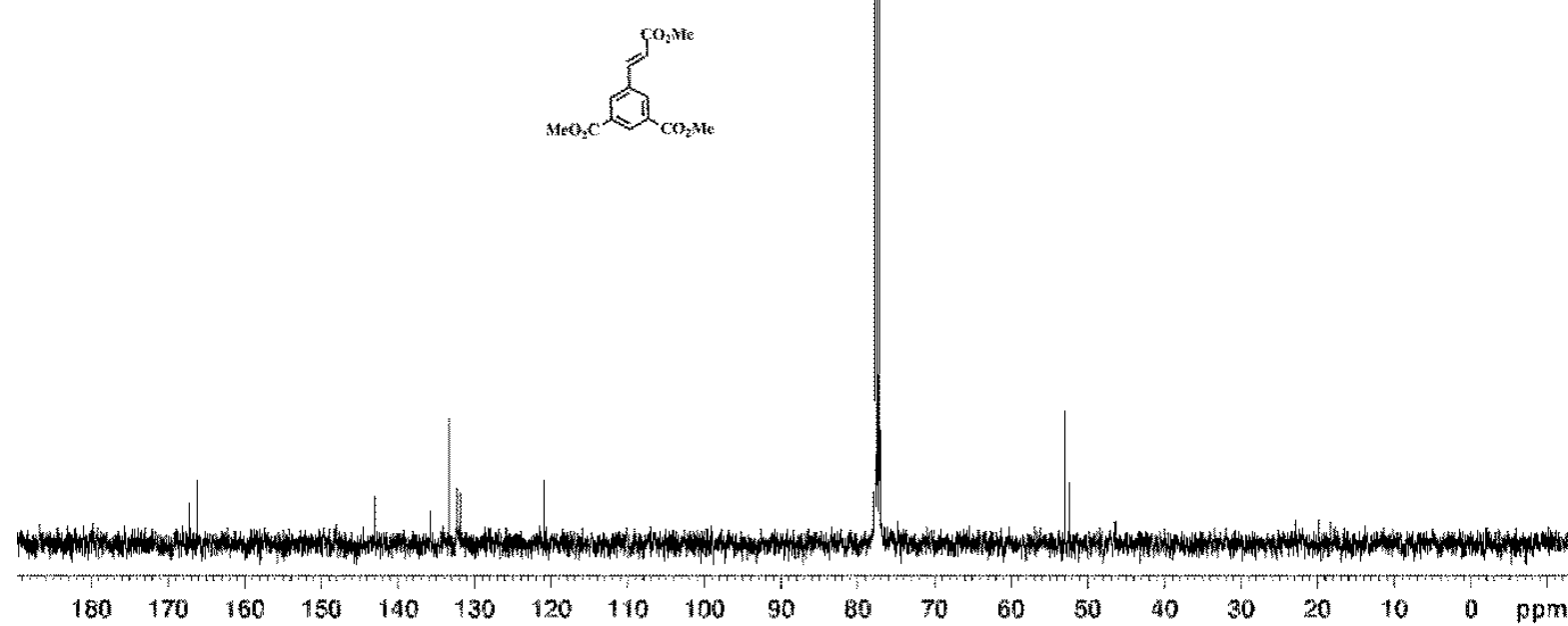



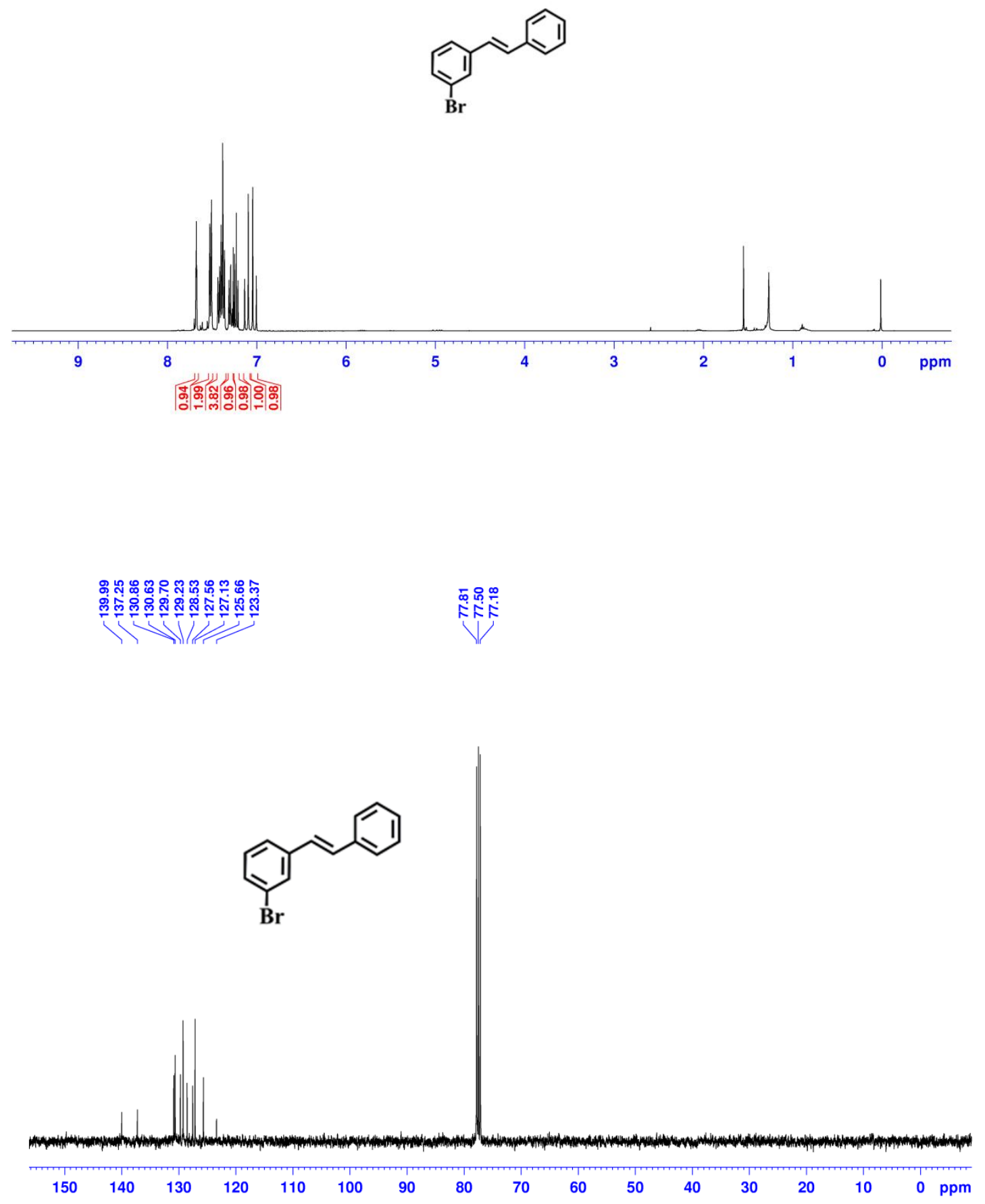

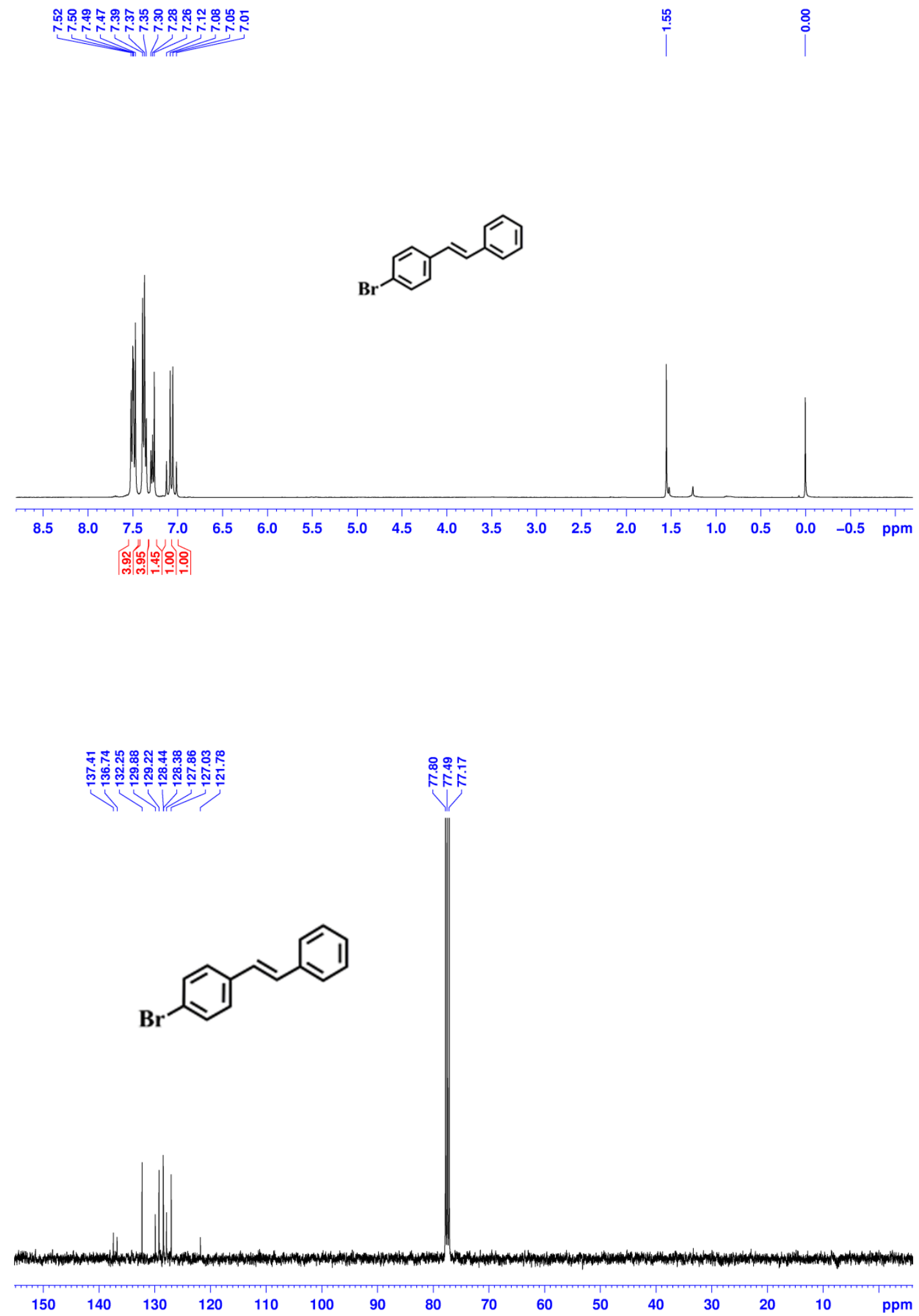

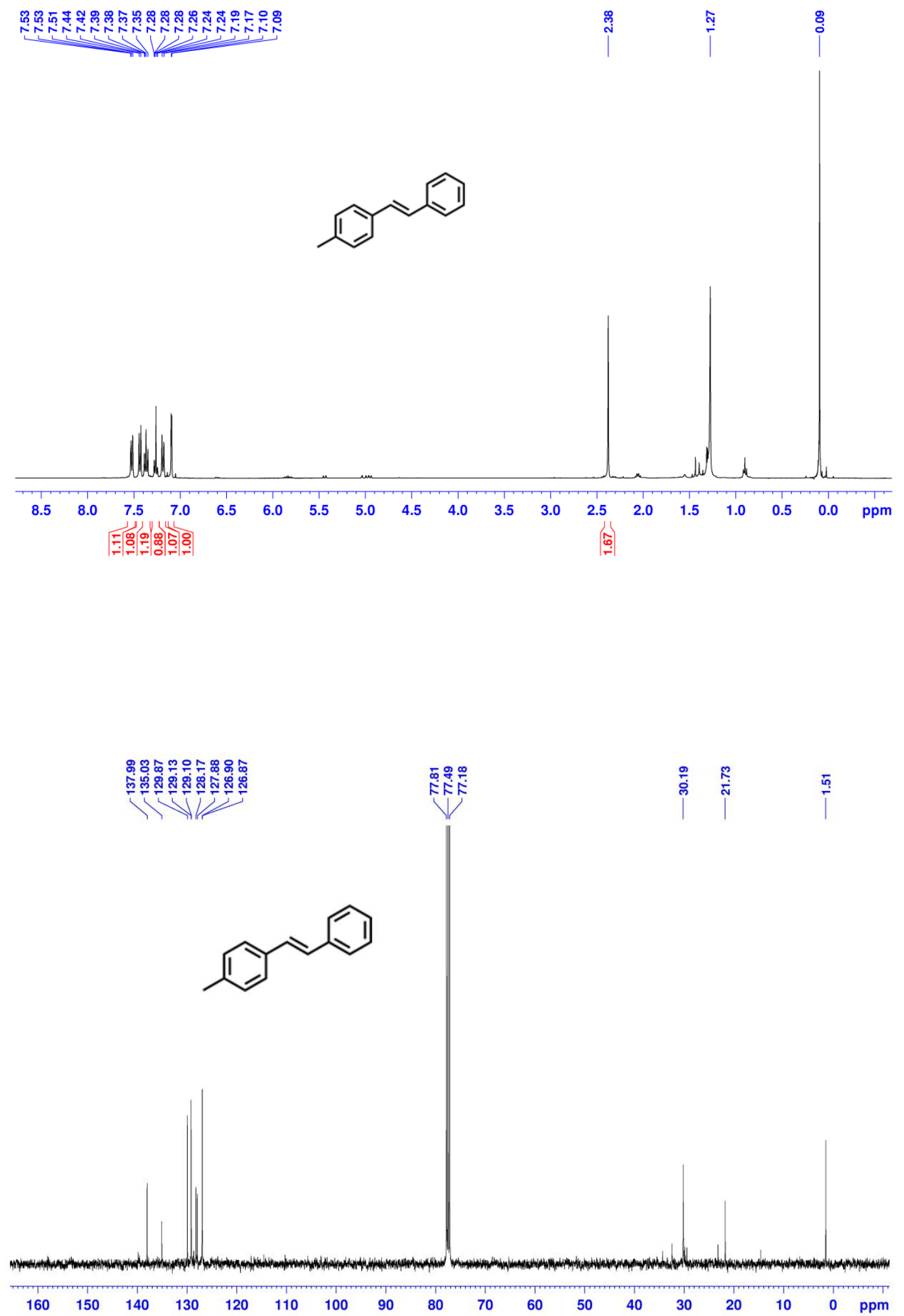


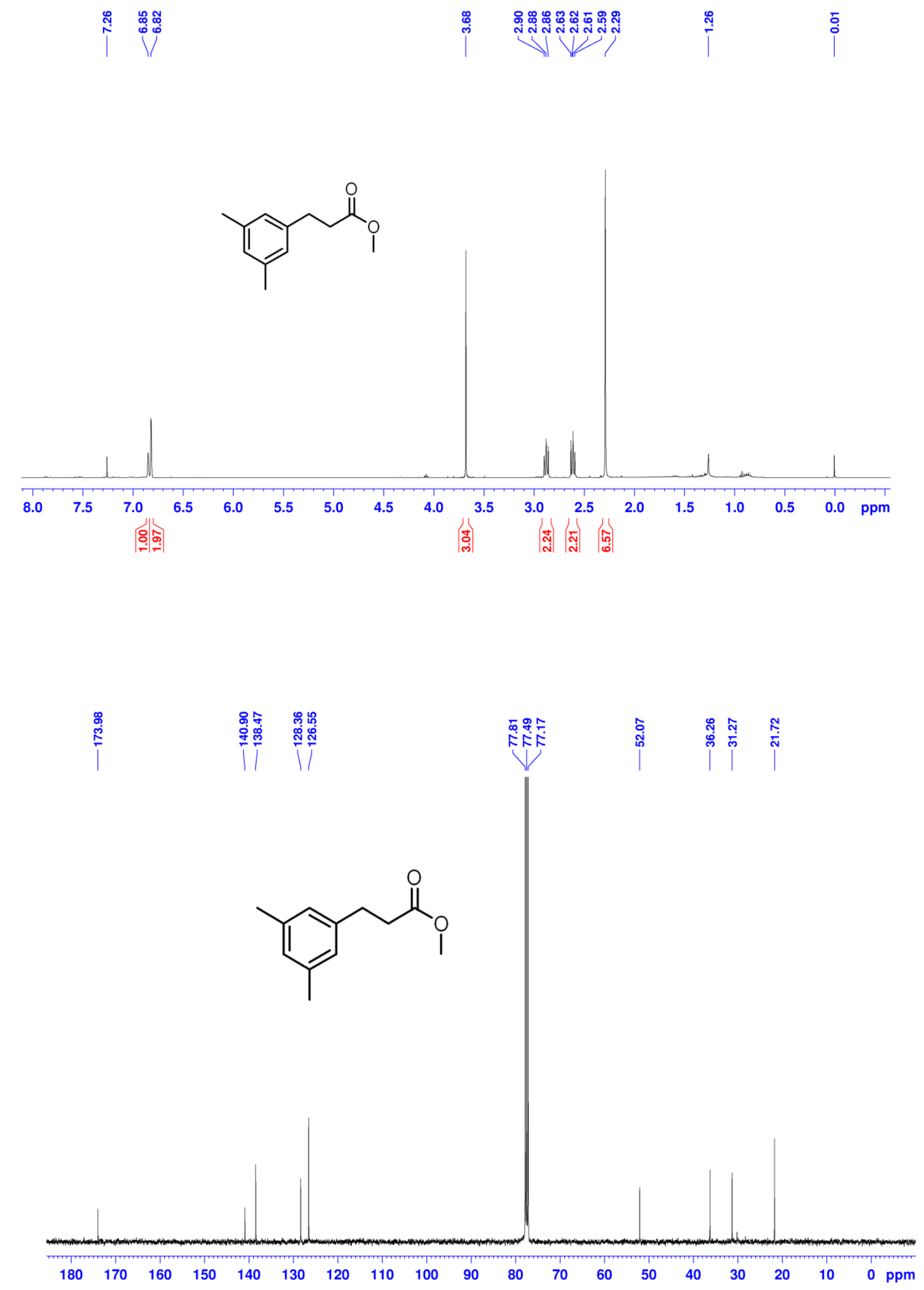




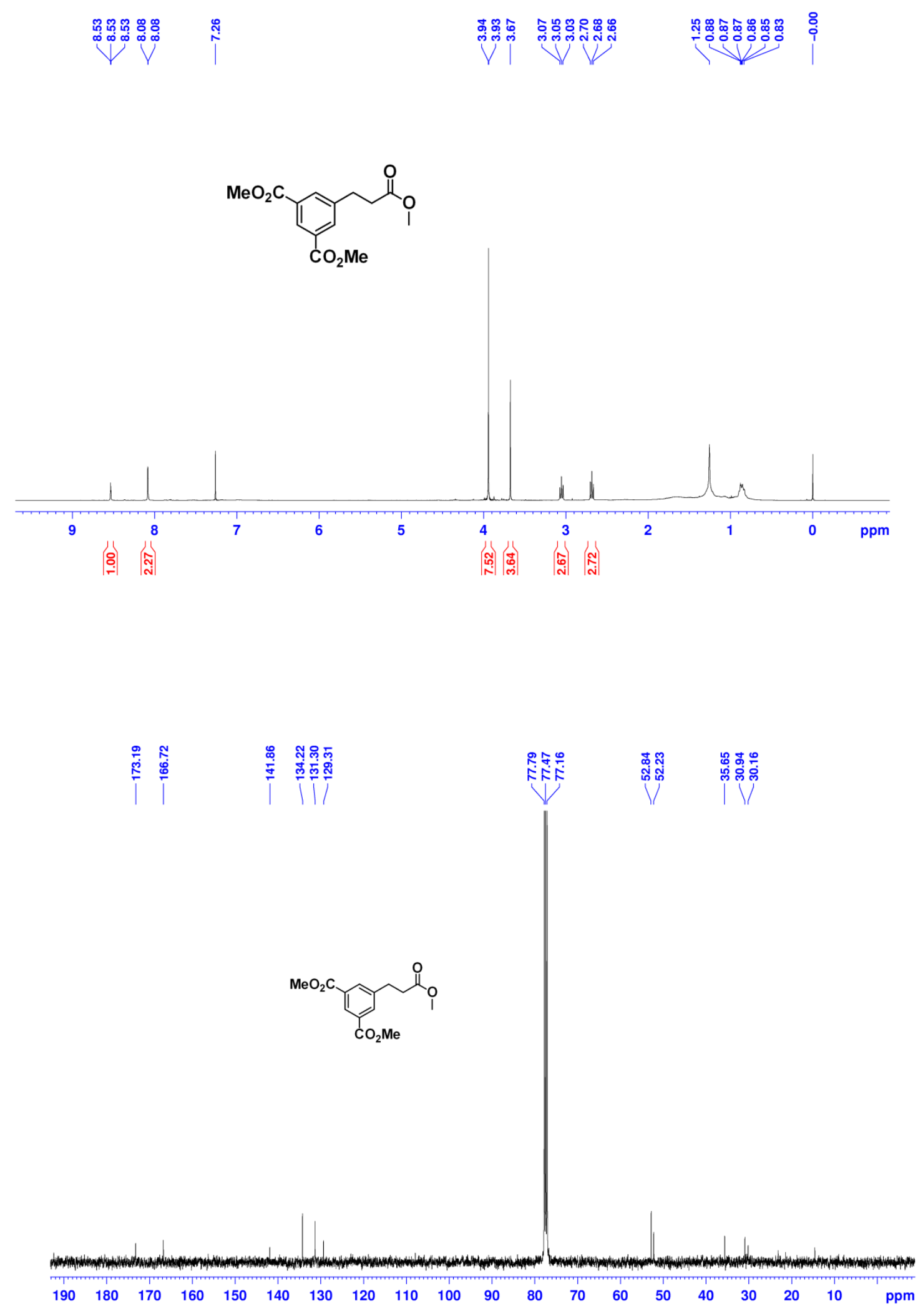



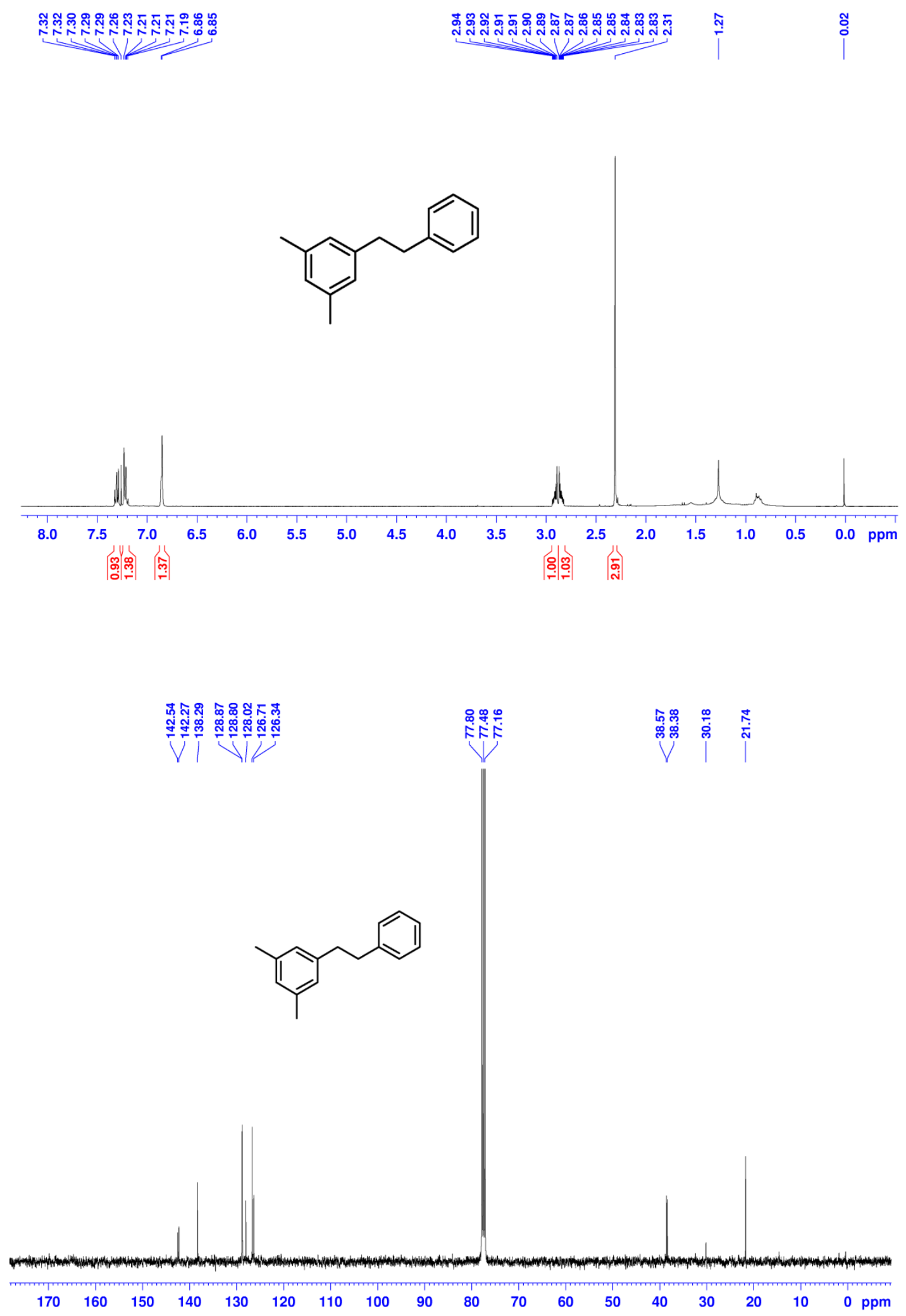

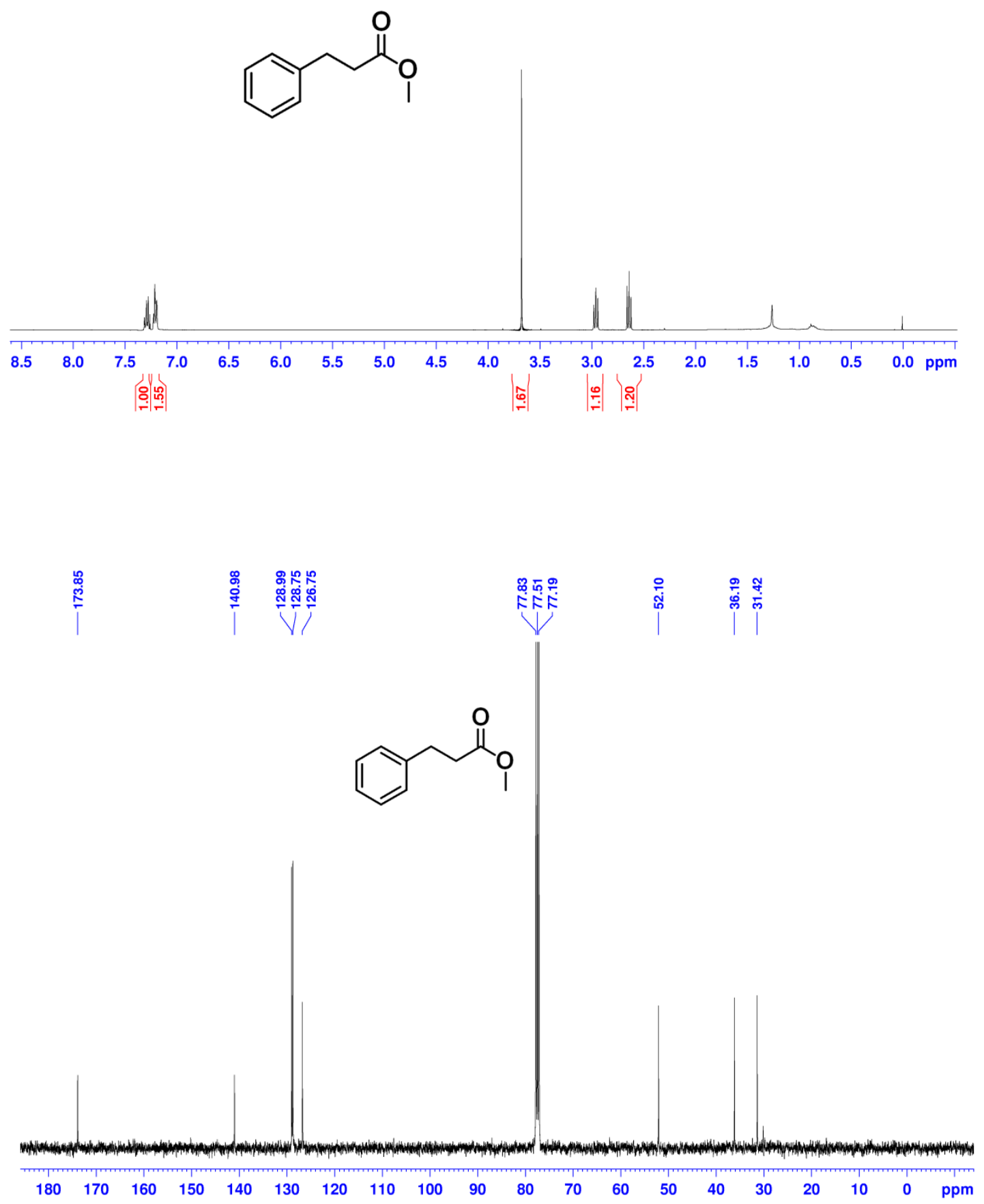


\section{References:}

(1) a) Faust, T. B.; Bellini, V.; Candini, A.; Carretta, S.; Lorusso, G.; Allan, D. R.; Carthy, L.; Collison, D.; Docherty, R. J.; Kenyon, J.; Machin, J.; Mclnnes, E. J. L.; Muryn, C. A.; Nowell, H.; Pritchard, R. G.; Teat, S. J.; Timco, G. A.; Tuna, F.; Whitehead, G. F. S.; Wernsdorfer, W.; Affronte, M.; Winpenny, R. E. P. Chem. Eur. J. 2011, 17, 14020-14030; b) Y.-L. Zhao, W. R. Dichtel, A. Trabolsi, S. Saha, I. Aprahamian, J. F. Stoddart, J. Am. Chem. Soc. 2008, 130, 11294-11296.

(2) Gole, B.; Bar, A. K.; Mukherjee, P. S. Chem. Eur. J. 2014, 20, 2276-2291.

(3) SMART/SAINT; Bruker AXS, Inc., Madison, WI, 2004.

(4) Sheldrick, G. M. SHELX-97, University of Göttingen: Göttingen, Germany, 1998.

(5) a) Farrugia, L. J. J. Appl. Crystallogr. 1999, 32, 837-838; bFarrugia, L. J. WinGX, version 1.65.04, Department of Chemistry, University of Glasgow: Glasgow, Scotland, 2003.

(6) Sheldrick, G. M. SADABS, University of Göttingen: Göttingen, Germany, 1999.

(7) Gole, B.; Sanyal, U.; Mukherjee, P. S. Chem. Commun. 2015, 51, 4872-4875. 